\title{
LG Solid Oxide Fuel Cell (SOFC) Model Development
}

\author{
Final Report
}

Report Period Start Date: October 1, 2009

Report Period End date: March 31, 2013

\author{
Principal Authors \\ Dr. Ben Haberman \\ Dr. Carlos Martinez-Baca \\ Greg Rush
}

Report Issue Date: May 31, 2013

DOE Award Number: DE-FE0000773

Submitting Organization

LG Fuel Cell Systems Inc.

(formerly known as Rolls-Royce Fuel Cell Systems (US) Inc.) (LGFCS)

6065 Strip Avenue NW

North Canton, $\mathrm{OH} 44720$ 
(C) 2013 LG Fuel Cell Systems Inc. This paper was written with support of the U.S. Department of Energy under Contract No. DE-FE0000773. The Government reserves for itself and others acting on its behalf a royalty-free, nonexclusive, irrevocable, worldwide license for Governmental purposes to publish, distribute, translate, duplicate, exhibit, and perform this copyrighted paper.

\section{Acknowledgment}

This material is based upon work supported by the Department of Energy National Energy Technology Laboratory under Award Number DE-FE0000773.

\section{Disclaimer}

This report was prepared as an account of work sponsored by an agency of the United States Government. Neither the United States Government nor any agency thereof, nor any of their employees, makes any warranty, express or implied, or assumes any legal liability or responsibility for the accuracy, completeness, or usefulness of any information, apparatus, product, or process disclosed, or represents that its use would not infringe privately owned rights. Reference herein to any specific commercial product, process, or service by trade name, trademark, manufacturer, or otherwise does not necessarily constitute or imply its endorsement, recommendation, or favoring by the United States Government or any agency thereof. The views and opinions of authors expressed herein do not necessarily state or reflect those of the United States Government or any agency thereof. 


\begin{abstract}
This report presents a summary of the work performed by LG Fuel Cell Systems Inc. during the project LG Solid Oxide Fuel Cell (SOFC) Model Development (DOE Award Number: DE-FE0000773) which commenced on October 1, 2009 and was completed on March 31, 2013. The aim of this project is for LG Fuel Cell Systems Inc. (formerly known as Rolls-Royce Fuel Cell Systems (US) Inc.) ("LGFCS") to develop a multi-physics solid oxide fuel cell (SOFC) computer code (MPC) for performance calculations of the LGFCS fuel cell structure to support fuel cell product design and development. A summary of the initial stages of the project is provided which describes the MPC requirements that were developed and the selection of a candidate code, STAR-CCM+ (CD-adapco). This is followed by a detailed description of the subsequent work program including code enhancement and model verification and validation activities. Details of the code enhancements that were implemented to facilitate MPC SOFC simulations are provided along with a description of the models that were built using the MPC and validated against experimental data. The modeling work described in this report represents a level of calculation detail that has not been previously available within LGFCS.
\end{abstract}




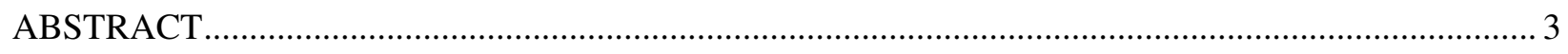

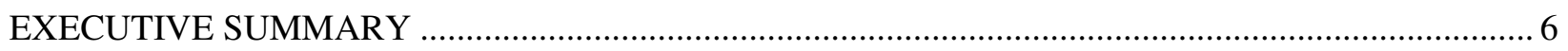

1 REVIEW OF LGFCS REQUIREMENTS AND EXISTING RESEARCH................................. 8

1.1 MODELING 8

$\begin{array}{ll}1.2 & 10\end{array}$

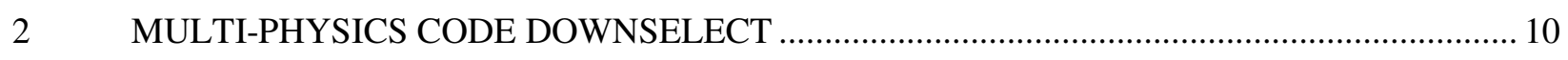

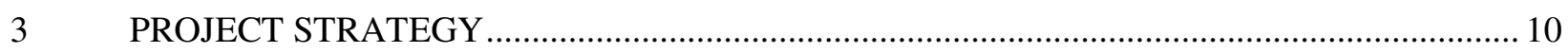

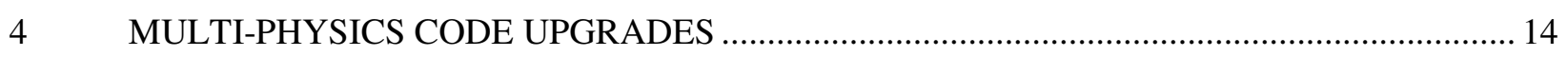

$\begin{array}{lll}4.1 & \text { FLUID FLOW MODELS } & 14\end{array}$

4.1.1 LOW FIDELITY 14

4.1.2 HIGH FIDELITY 14

4.2 POROUS FLOW MODELS 15

$\begin{array}{lll}4.2 .1 & 15\end{array}$

$\begin{array}{lll}\text { 4.2.2 HIGH FIDELITY } & 16\end{array}$

$\begin{array}{lll}4.3 & \text { THERMAL MODELS } & 18\end{array}$

$\begin{array}{lll}4.3 .1 & \text { LOW FIDELITY } & 18\end{array}$

$\begin{array}{lll}\text { 4.3.2 HIGH FIDELITY } & 18\end{array}$

$\begin{array}{lll}4.4 & \text { CHEMISTY MODELS } & 18\end{array}$

4.4.1 LOW FIDELITY 18

4.4.2 HIGH FIDELITY 19

$\begin{array}{lll}4.5 & \text { CURRENT FLOW MODELS }\end{array}$

$\begin{array}{lll}\text { 4.5.1 LOW FIDELITY } & 20\end{array}$

4.5.2 HIGH FIDELITY 22

4.6 ELECTROCHEMISTRY MODELS 22

$\begin{array}{lll}\text { 4.6.1 LOW FIDELITY } & 22\end{array}$

4.6.2 HIGH FIDELITY 25

4.7 DEGRADATION MODELS 26

$\begin{array}{lll}\text { 4.7.1 LOW FIDELITY } & 26\end{array}$

$\begin{array}{lll}\text { 4.7.2 HIGH FIDELITY } & 28\end{array}$

$\begin{array}{lll}4.8 & \text { TIME MODEL } & 28\end{array}$

$\begin{array}{lll}4.8 .1 & \text { LOW FIDELITY } & 28\end{array}$

4.8.2 HIGH FIDELITY 29

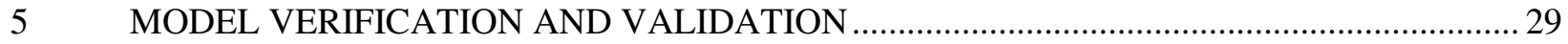

$\begin{array}{lll}5.1 & \text { SINGLE CELL MODEL VALIDATION } & 30\end{array}$

5.2 PENTA CELL MODEL VALIDATION 34

5.3 SUBSTRATE MODEL VALIDATION 239

$5.4 \quad$ BUNDLE MODEL VALIDATION $\quad 42$

5.5 LOW FIDELITY EMPIRICAL DEGRADATION MODEL VALIDATION 44

5.6 HIGH FIDELITY KINETIC DEGRADATION MODEL DEMONSTRATION 45

5.7 DEMONSTRATION OF MPC TRANSIENT CAPABILITIES (LARGE TIME STEPS) 45

5.8 DEMONSTRATION OF MPC TRANSIENT CAPABILITIES (SMALL TIME STEPS) 48

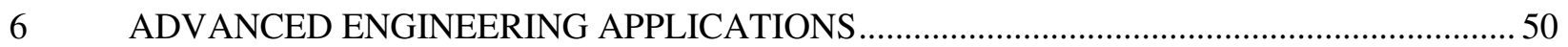

$6.1 \quad$ STRIP FUEL DISTRIBUTION 250

6.2 DETAILED ANALYSIS OF SUBSTRATE ELECTRICAL CONNECTIONS 51 


\begin{tabular}{|c|c|}
\hline 6.3 & THERMAL TRANSIENT ANALYSIS \\
\hline 6.4 & THERMAL ANALYSIS OF BUNDLE OPERATION WITHIN A BLOCK \\
\hline 6.5 & DETAILED ANALYSIS OF BUNDLE ELECTRICAL CONNECTIONS \\
\hline 6.6 & DETAILED GEOMETRIC MODELING OF CELL COMPONENTS \\
\hline 7 & TESTING OF MPC WITH ENGINEERING STAFF... \\
\hline 8 & CONCLUSION... \\
\hline 9 & LIST OF FIGURES . \\
\hline 10 & REFERENCES ..... \\
\hline & LIST OF ACRONYMS AND ABBREVIATIONS. \\
\hline
\end{tabular}




\section{EXECUTIVE SUMMARY}

The final report for contract DE-FE0000773 summarizes project activity from the contract start on October 1, 2009 until the scheduled completion date of March 31, 2013. This completion date followed approval of two no-cost project extensions of one year and six months respectively. At completion of the contracted workscope, LG Fuel Cell Systems (LGFCS) has developed a multi-physics solid oxide fuel cell (SOFC) computer code for performance analysis of the LGFCS fuel cell structure. This analysis capability will continue to support fuel cell product design and development. Further, this work consolidates years of LGFCS investment and research throughout the world as part of its business objective to develop an SOFC product for stationary power generation.

The planned work for this project consisted of:

i) Initial code downselect activity

ii) Review and consolidation of prior LGFCS fuel cell modeling capabilities into the multiphysics computer code (MPC)

iii) Eight subsequent code releases to implement enhancements for SOFC simulations

iv) Code release validation against experimental data

The downselect activity identified STAR-CCM+ (CD-adapco) as the best candidate for the MPC. Code Release 1 was completed on schedule during the first project year and Code Releases 2 and 3 were completed in the second project year. The third project year involved the completion of Code Release 4 (originally scheduled for project year 2) and work for Code Releases 5 through 8, the latter two being completed in project year 4 .

Project activity in the first project year (10/1/09-9/30/10) commenced with research reviews and the multi-physics code downselect. An extensive review of published SOFC modeling research was initiated as well as an in-house review with internal LGFCS stakeholders to determine key MPC functional requirements (Milestone 1). These requirements guided the selection of candidate MPC codes for evaluation and the development of extensive tests for a vendor evaluation exercise. This exercise was completed as scheduled and the test scores indicated that STAR-CCM+ (CD-adapco) was the preferred multi-physics code and this choice was widely discussed and approved, leading to the successful completion of Milestone $2(1 / 1 / 2010)$. During this time a project strategy was developed to leverage the custom modeling capabilities available within STAR-CCM+ to implement the required MPC code enhancements and ensure that the MPC was suitable for use by engineers outside of the project team. This strategy involved storing model data in a Microsoft Excel spreadsheet and automating all model processes (including the installation of code enhancements) using a custom made Graphical User Interface (GUI) plug-in installed into STAR-CCM+.

Project activities for Code Release 1 commenced at the start of 2010 and involved the implementation of the code enhancements necessary to build a basic single fuel cell model and its validation against single cell experimental test data. Code enhancements for modeling porous flows and electrochemistry and existing STAR-CCM+ models for fluid flow and chemistry were implemented into an early version of the GUI. A one-dimensional single cell model was successfully validated against single cell experimental test data, leading to the completion of Code Release 1 (Milestone $3-5 / 31 / 10$ ).

The work plan for Code Release 2, which commenced on time (6/1/10), focused on implementing code enhancements for current flow modeling and the extension of model verification/validation to two and three-dimensional models of five-cell (penta cell) and substrate sized test pieces. Code enhancements were added into the GUI to enable the numerical simulation of heat transfer, current flow and the 
integration of the current flow and electrochemistry models. Validation and verification tasks included the completion of penta cell model validation (two and three-dimensional models) and the building of a three-dimensional substrate model. Code Release 2 (Milestone 4) was scheduled for completion in the second project year (10/1/10-9/30/11) and slipped from November 2010 to January 2011 because of priority code analysis work needed to support other activities in the LGFCS business.

Project activities for Code Release 3 commenced at the start of 2011 and focused on the validation of the substrate model, development of empirical degradation models for cell durability, and development of a bundle scale model. Additional MPC features were also developed to support construction of the large models and included automated mesh generation and an electrical network model to facilitate the electrical connection between rows of cells in a bundle. Code Release 3 (Milestone 5) was completed on time in June 2011.

Code Release 4 commenced on time June 2011 and focused on validating the bundle model against experimental measurements and extending the empirical degradation model to incorporate kinetic rate calculations. Additional MPC features were also developed to improve model run times on parallel computer processors and these included an acceleration algorithm and an improved model configuration from imported CAD geometry. Code Release 4 (Milestone 6) was completed in November 2011 during the third project year (10/1/11-9/30/12) following a two month schedule slip which resulted from the need to develop an acceleration algorithm to significantly improve bundle model convergence time and also the need to support other high priority LGFCS CFD analysis.

Code Release 5 involved the implementation of the MPC transient simulation capability for large time steps (which required the reorganization of the MPC to support time dependent analysis) and the testing of transient calculations on a range of models from single cell to bundle scales. Code Release 5 (Milestone 7) commenced in November 2011 and was completed in January 2012.

Code Release 6 was focused on the testing of MPC capabilities in advanced engineering applications and the extension of the MPC transient capabilities to very small time steps. Comparisons with predictions from other codes were also made to verify the accuracy of the MPC models and their implementation. Additional MPC features were developed as a result of feedback from Code Release 5 activities and these included improvements to the porous flow model, to the stability of electrochemistry calculations during the onset of fuel starvation and to parallel computation efficiency. Code Release 6 (Milestone 8) commenced in February 2012 and was completed in June 2012.

The planned activities for Code Release 7 included the enhancement of the GUI and completion of MPC documentation in order to prepare the MPC for use by engineering staff outside of the project team. Existing MPC features were further enhanced as a result of feedback from Code Release 6 activities and these included improvements to the MPC porous flow and gas mixture viscosity models. Code Release 7 (Milestone 9) commenced in October 2011 and was completed in March 2013.

Code Release 8 focused on the testing of MPC capabilities in further advanced engineering applications which included highly detailed modeling of cell layout and further validation of MPC model predictions against experimental data. Enhancements were also made to the automated process for importing CAD model geometry following suggestions from $\mathrm{CD}$-adapco staff and to the modeling of current flow to allow more realistic cell geometries to be considered. Code Release 8 (Milestone 10) commenced in July 2012 and was completed in March 2013. 
The purpose of this project was for LG Fuel Cell Systems (LGFCS) to develop a solid oxide fuel cell (SOFC) multi-physics computer code (MPC) for performance analysis of the LGFCS fuel cell structure to support fuel cell product design and development. The following requirements for the development of the MPC were formulated in consultation with LGFCS staff and through an extensive review of published research and in-house expertise. This review and consultation exercise (Milestone 1) was successfully completed on schedule in 2009.

The MPC is intended for use by LGFCS staff in a wide variety of applications including the simulation of fuel cell performance, analyzing fluid flow in peripheral components, and providing temperature distributions to stress and reliability predictive models. Typical fuel cell applications range from simulating the performance of a single cell, see Figure 1(a), to a complete substrate including a row of cells fabricated on each side, see Figure 1(c), and a bundle consisting of six substrates, see Figure 1(d).

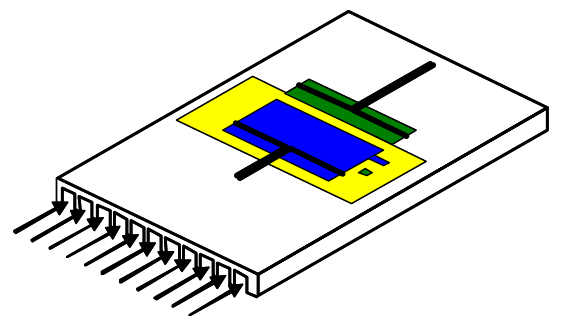

(a) Single cell test piece

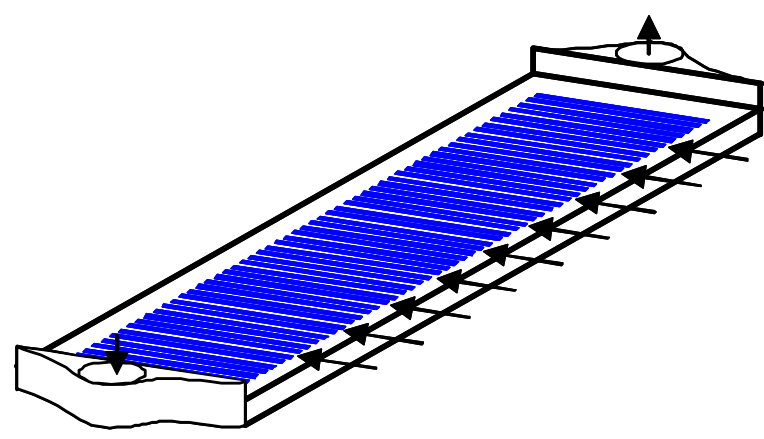

(c) Substrate

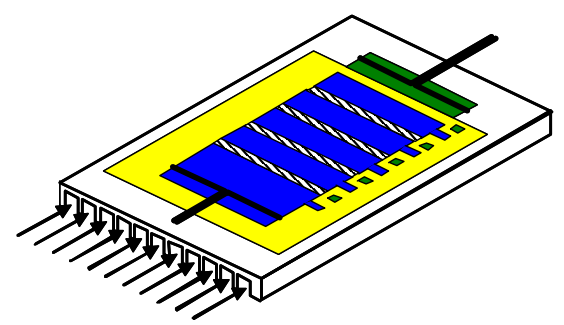

(b) Five cell test piece

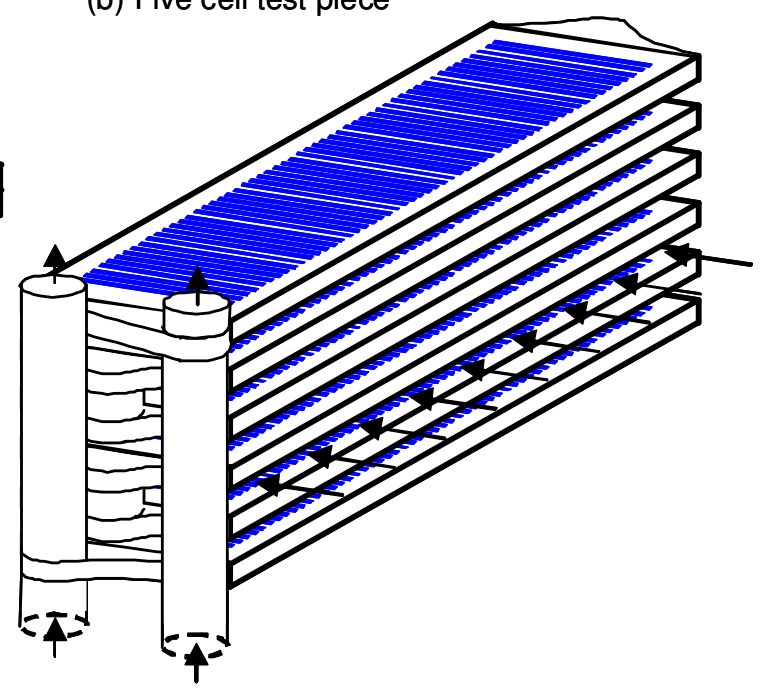

(d) Bundle

Figure 1 Examples of model geometry.

\subsection{MODELING}

The requirements for modeling these SOFC applications include a wide range of physical phenomena:

- Fluid Flow which can be laminar or turbulent, steady-state or transient, and describes the transport of fuel through manifolds and along internal substrate channels with air flow over the outside of the substrates. 
- Porous Flows which describe the transport of gases through porous components such as the substrate and the fuel cell electrodes.

- Heat Transfer by convection, conduction and radiation which determine the temperature distribution throughout a model.

- Chemical reactions which are catalyzed by the fuel cell components and make significant contributions to fuel cell behavior.

- Electrochemistry which governs the behavior of the charge transfer reactions occurring at the interfaces between the cell electrodes and the electrolyte.

- Current Flow including the flow of electrical current through the fuel cell electrodes and the electrical connections between substrates in addition to the ionic current flow in the electrolytes.

- Degradation which reduces cell performance over time via physical and chemical processes.

The inclusion of the SOFC physics mentioned above is sufficient to enable the MPC to make accurate simulations of the variety of non-SOFC applications required by LGFCS. The following more general requirements were identified by LGFCS staff during the review exercise:

- The MPC must solve the above physical models on realistic geometry which has been imported directly from CAD geometry data.

- Solution of steady state and transient problems over a range of time scales.

- A degree of modeling flexibility is required to allow application dependent model customization. For example, a one-dimensional isothermal model is sufficient for single cell calculations whereas three-dimensional thermal models are a necessity for making accurate bundle simulations.

- The MPC must provide a user interface such that it is possible for LGFCS staff outside of the code development team to efficiently build and run simulations.

Model flexibility is achieved by providing interchangeable high and low fidelity versions of each physical model as shown in Table 1.

Table 1 High and low fidelity models.

\begin{tabular}{|l|l|l|}
\hline \multirow{2}{*}{ Physical Model } & \multicolumn{2}{|c|}{ Low } \\
\hline Fluid Flow & $\begin{array}{l}\text { 1D plug flows } \\
\text { (Steady-state, laminar) }\end{array}$ & \multicolumn{1}{c|}{ CFD calculations } \\
\hline Porous Flow & Darcy model & CPIM model \\
\hline Heat Transfer & Isothermal & $\begin{array}{l}\text { Convection, conduction and } \\
\text { radiation }\end{array}$ \\
\hline Chemistry & Global one step reactions & Multi-step reactions \\
\hline Electrochemistry & Uniform current density & $\begin{array}{l}\text { Integrated with current flow } \\
\text { simulation }\end{array}$ \\
\hline Current Flow & Analytical ohmic loss model & 3D numerical model \\
\hline Degradation & Empirical models & Kinetic models \\
\hline Time & Steady-state & Transient \\
\hline
\end{tabular}




\subsection{INTERACTIONS}

In addition to providing a detailed simulation capability for LGFCS the MPC is also required to support other projects within the company. These interactions include:

- Supporting lifetime and reliability predictions which will require detailed temperature distributions from substrate and bundle simulations.

- Providing accurate validated models of fuel cell processes, such as electrochemistry, in a format that is suitable for use by larger less detailed models.

\section{MULTI-PHYSICS CODE DOWNSELECT}

Three candidate codes were chosen for evaluation as potential MPCs: FLUENT (ANSYS), STAR-CCM+ (CD-adapco) and MARC (MSC software). A set of code evaluation criteria was created based on the MPC requirements described in Section 1 and a series of tests were developed to measure the performance of each code against these criteria. These test questions were passed to each code vendor and two of the three vendors (ANSYS and CD-adapco) were able to provide results in the time available. The test results were scored against the evaluation criteria to give an overall performance measure for each candidate code. The evaluation exercise was completed as scheduled at the end of November 2009.

The vendor test results and evaluation scores that were obtained during the evaluation exercise were reviewed within LGFCS and Rolls-Royce plc during December 2009. The test scores indicated that STAR-CCM+ (CD-adapco) was the preferred multi-physics code and this choice was extensively discussed and approved. The overall findings of the evaluation and downselect exercises were presented to the DOE and approved before the end of 2009, which lead to the successful completion of Milestone 2 on January 1, 2010.

\section{PROJECT STRATEGY}

STAR-CCM+ has a number of useful features that were identified during the downselect to support the LGFCS simulation requirements:

- Advanced physical modeling capabilities that allowed much of the MPC project requirements to be met through existing code features.

- Highly advanced geometric modeling features including, import from external CAD files, built in CAD modeling and highly flexible meshing tools.

- Customization capabilities that allowed user defined functions to be incorporated into the conservation equations being solved.

- Automation capabilities that allowed tasks to be performed automatically and remotely using Java macros and plug-ins that could be installed directly into the STAR-CCM+ Graphical User Interface (GUI).

The MPC project strategy involves leveraging these features to automate the tasks necessary to build and run simulations for the applications required by LGFCS as shown in Figure 2. 


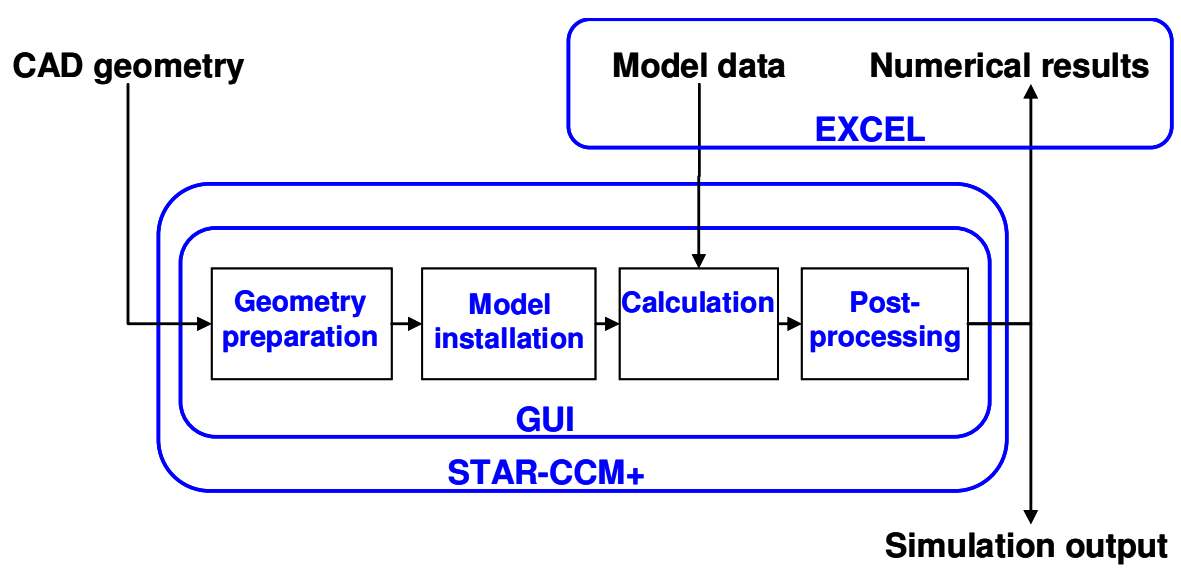

Figure 2 The MPC components.

As Figure 2 shows the MPC consists of several distinct components:

- $\quad$ STAR-CCM+ the CFD software package on which the MPC is based.

- A Microsoft Excel model spreadsheet (EMS) that is used to store all numerical model inputs and outputs.

- A graphical user interface (GUI) installed into STAR-CCM+ allows the user to perform a series of automated tasks to pre-process, execute and post-process simulations 
The EMS is used as a convenient means to store model inputs and some selected numerical output from simulations. The EMS has several worksheets and a typical view is shown below in Figure 3 which illustrates some of the fields (highlighted in yellow) that are available for user input.

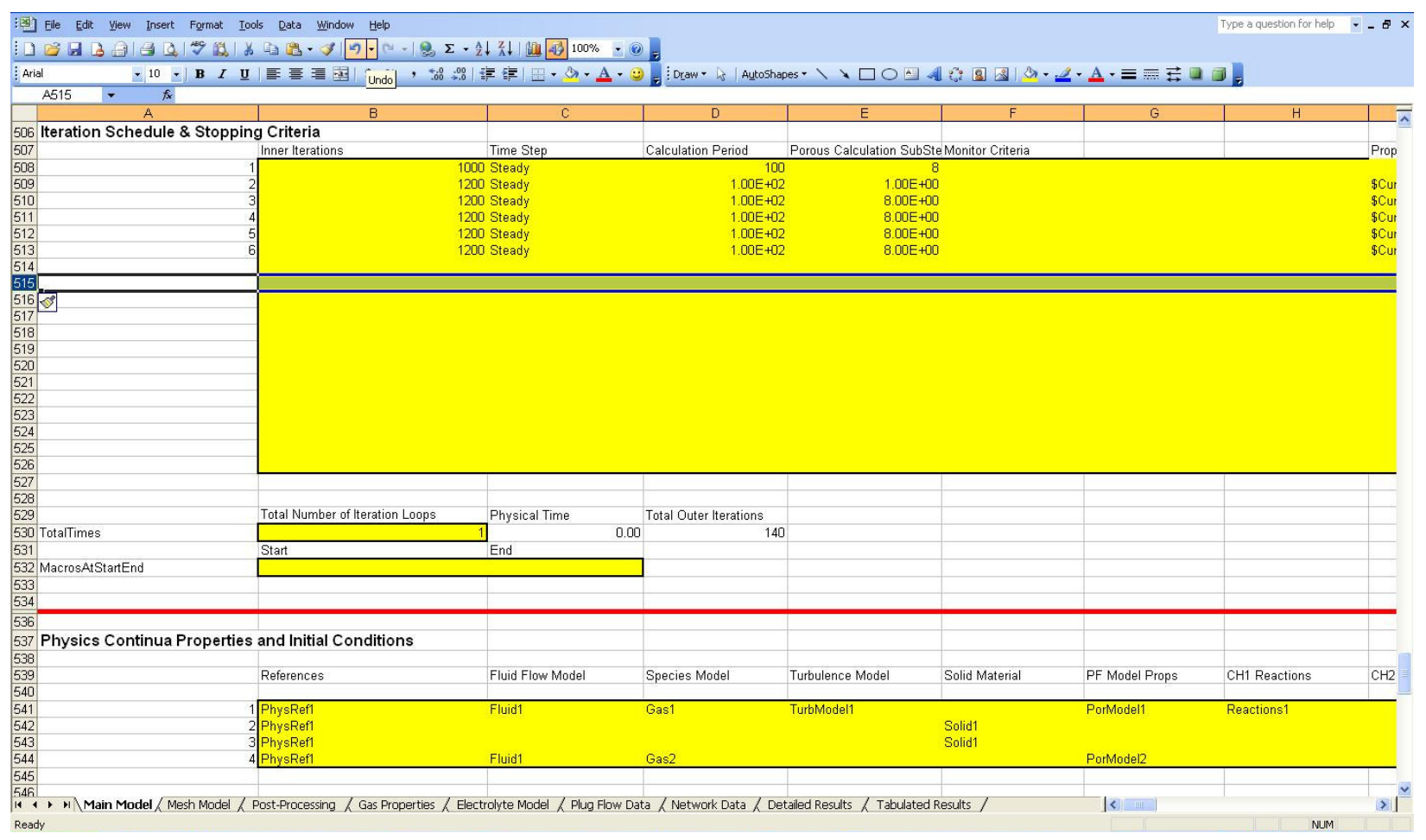

Figure 3 A typical view from the Excel model spreadsheet (EMS)

The GUI is installed into STAR-CCM+ as a plug-in and provides an interactive environment to perform a range of automated tasks required to pre-process, execute and post-process a simulation. The MPC GUI is built on the Java NetBeans platform [3] which is also used to build the STAR-CCM+ GUI. Figure 4 shows a typical view of the GUI within STAR-CCM+ and the salient features of GUI operation within STAR-CCM+. 


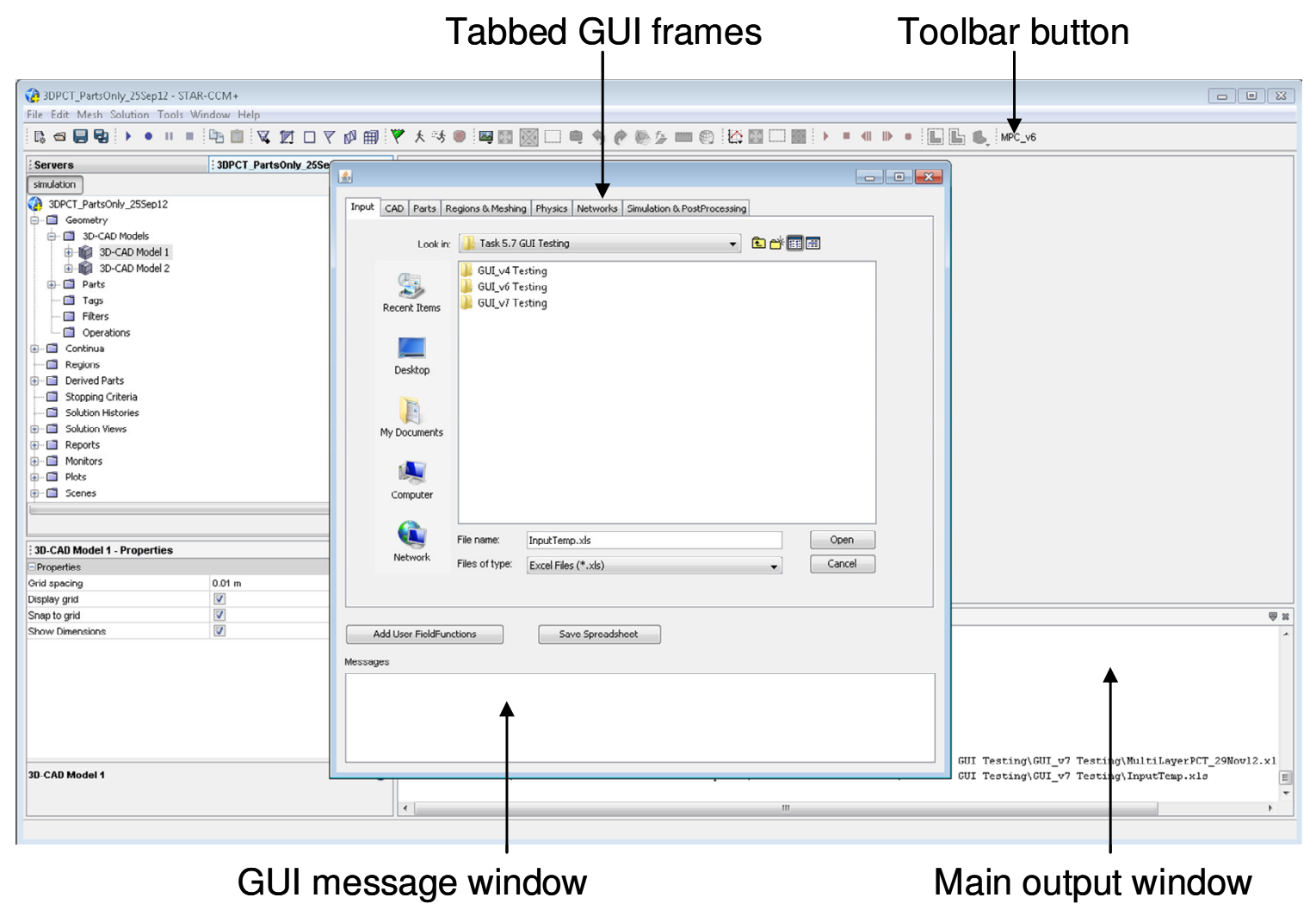

Figure 4 A typical view of the GUI installed into STAR-CCM+.

The main GUI features are as follows:

- Once installed the GUI is activated via a toolbar button

- The GUI has several tabbed pages that provide selections of tools appropriate for different stages within the overall MPC workflow.

- Layout has been arranged to provide a step by step approach to model building.

- Each page contains a series of buttons that activate different tasks - taking inputs from the EMS and user selection windows which are available on some of the pages.

- The GUI has its own message window that provides a summary of any errors and useful tips when a task is performed.

- Each task performed generates more detailed output in the main STAR-CCM+ output window

Each task executes Java code and can perform several functions:

- Controlling operations within STAR-CCM+ to automatically perform tasks that would otherwise be performed manually.

- Reading and writing data to the EMS.

- Performing additional calculations that are not part of STAR-CCM+. 
- Providing user feedback via the GUI message window and the main STAR-CCM+ output window on the progress of the task being performed. If a task fails then error messages explaining why and useful tips are given to suggest how to fix any problems.

The features described above provide the MPC with the functionality to automatically perform the tasks required to take a model through the STAR-CCM+ workflow and generate SOFC and other simulations. The GUI has been specifically designed to meet the MPC project objective of providing SOFC simulation capability to engineering staff outside of the project team.

\section{MULTI-PHYSICS CODE UPGRADES}

A wide range of code developments have been implemented into the MPC and made available through the eight planned Code Releases. As mentioned in Section 1, a high and low fidelity version of each physical model is provided to facilitate application dependent model customization, see Table 1 . These physical models are described in the following subsections.

\subsection{FLUID FLOW MODELS}

These models form the basis of the MPC physical modeling capability and are used to describe the transport of a fluid comprising a mixture of gases through all fluid and porous model components.

\subsubsection{LOW FIDELITY}

The low fidelity fluid flow model is used to improve accuracy when a very coarse computational mesh is being used to describe fluid flow through pipes and ducts. This model has the following features:

- Applicable to long straight pipes or internal channels where flow is steady-state and fullydeveloped.

- Requires the installation of high fidelity fluid flow model.

- User supplied pipe or duct aspect ratio is used to evaluate expected pressure gradient in pipe.

- Additional friction term added to correct pressure gradient and improve the accuracy of the numerical predictions resulting from using a coarse computational mesh.

\subsubsection{HIGH FIDELITY}

The high fidelity fluid flow model utilizes default STAR-CCM+ CFD capabilities with some MPC improvements and has the following features:

- Steady-state laminar and turbulent fluid flow described by the Navier-Stokes equations.

- Flow geometry that can be two or three-dimensional.

- Multi-component gas mixture with uniform species diffusivities.

- Constant density or compressible ideal gases.

- Constant gas species thermal conductivity, a mixture value is obtained through mole fraction averaging.

- Gas species viscosity can be constant or $6^{\text {th }}$ order polynomial in temperature, mixture values can be mole or mass fraction averages. 
- Thermodynamic properties of gas components described by NASA database which provides $6^{\text {th }}$ order polynomial fits for temperature dependent properties such as specific heat capacity and enthalpy.

\subsection{POROUS FLOW MODELS}

The porous flow models are used to enhance the fluid flow models with improved calculations to model the effects of porous materials and multi-component diffusion in a mixture of gases.

\subsubsection{LOW FIDELITY}

The low fidelity porous flow model enhances the default STAR-CCM porous flow capabilities and has the following features:

- Flow in porous materials is modeled assuming continuum physics.

- The viscous resistance for porous regions can be calculated assuming a capillary of specified diameter or prescribed directly.

- The inertial resistance for porous regions can be prescribed directly.

- Resistances can be anisotropic or isotropic.

- Gas species diffusivities are calculated individually using a binary mixture approximation.

Using this model enhances the diffusion capabilities of the high fidelity fluid flow modeling in both fluid and porous regions.

The format of the Darcy equation required by STAR-CCM+ is

$\nabla p=-\mathbf{P} \cdot \mathbf{U}$

where $\mathbf{P}$ is the viscous resistance vector term $\left(\mathrm{kg} \mathrm{m}^{-3} \mathrm{~s}\right)$. For a capillary resistance calculation $\mathbf{P}$ is given by

$\mathbf{P}=\mathbf{F} \frac{C_{t} \tau^{2}}{\varepsilon} \frac{32 \mu_{g}}{d_{p}{ }^{2}}$

where $\mu_{\mathrm{g}}$ is the dynamic viscosity of the gas mixture $\left(\mathrm{kg} \mathrm{m}^{-1} \mathrm{~s}^{-1}\right), d_{p}$ is the mean pore diameter $(\mathrm{m}), \tau$ and $\varepsilon$ are the tortuosity and porosity of the porous material respectively and $C_{t}$ is a thickness correction factor and $\mathrm{F}$ is a user defined vector of resistance multipliers which allows the specification of anisotropic material properties.

$C_{t}$ is used to account for two and three dimensional effects in one-dimensional models. For example, the webs between fuel channels increase the flow path to some parts of a fuel cell and this tends to increase the overall effective thickness of the tube $\left(C_{t}>1\right)$. In addition, the gaps between fuel cells tend to improve gas flow which effectively decreases the thickness of a tube $\left(C_{t}<1\right)$.

The mixture diffusivity approximation for each gas species diffusivity $D_{i}$ in a mixture of $N$ gases is given by 


$$
D_{i}=\frac{1}{C_{t}} \frac{\varepsilon}{\tau^{2}} \frac{1-X_{i}}{\sum_{j=1, j \neq i}^{N} X_{j} / D_{i j}}\left(\mathrm{~ms}^{-2}\right)
$$

where $X_{i}$ is the mole fraction of gas species $i$ and $D_{i j}$ is the continuum binary diffusion coefficient $\left(\mathrm{ms}^{-2}\right)$.

$D_{i j}$ are calculated for each pair of gases using the expression of Fuller et al. [1]

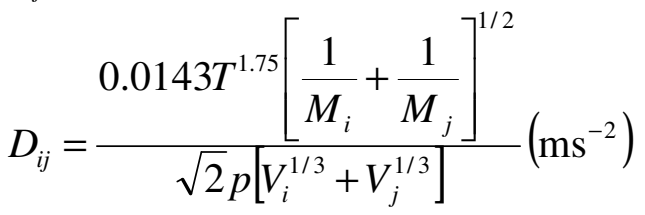

where $M_{i}$ is the molar mass $\left(\mathrm{kg} \mathrm{kmol}^{-1}\right)$ of gas species $i, T$ is the temperature $(\mathrm{K}), p$ is the pressure (Pa) and $V_{i}$ and $V_{j}\left(\mathrm{~m}^{3}\right)$ are the "Fuller" diffusion volumes [1].

\subsubsection{HIGH FIDELITY}

The high fidelity porous flow model enhances the default STAR-CCM porous flow capabilities and has the following features:

- Porous flows are modeled using the Cylindrical Pore Interpolation Model (CPIM) equations which provide a complete description of multi-component gas flows along porous capillaries [1].

- Continuum and non-continuum (Knudsen) effects and diffusion slip are accounted for.

- Enhanced gas diffusivity calculation in fluid regions using the Stefan-Maxwell equations which improves the capability of the high fidelity fluid flow model.

The CPIM equations in terms of the total area averaged mass fluxes of each gas species are:

$$
\begin{aligned}
& \nabla Y_{i} \rho_{g} \frac{\varepsilon}{\beta^{2}} \frac{1}{C_{t}}=\sum_{j=1, j \neq i}^{N}\left(\frac{\mathbf{G}_{j} Y_{i}}{D_{j i}^{e}}-\frac{\mathbf{G}_{i} Y_{j}}{D_{i j}^{e}}\right) \\
& \nabla p \frac{\varepsilon}{\beta^{2}} \frac{1}{C_{t}}=-A \sum_{j=1}^{N} \mathbf{G}_{j} / \sqrt{M_{j}}
\end{aligned}
$$

Where $D_{j i}^{e}, D_{i j}^{e}$ and $A$ are interpolated coefficients, which are given by

$$
\frac{1}{D_{i j}^{e}}=\frac{1}{D_{i j}}+\frac{1}{D_{k, i}}, \quad \frac{1}{D_{j i}^{e}}=\frac{1}{D_{j i}}+\frac{1}{D_{k, j}}, \quad \frac{1}{A}=\frac{1}{A^{0}}+\frac{1}{A^{\infty}}
$$

and $M_{i}$ is the molar mass $\left(\mathrm{kg} \mathrm{kmol}^{-1}\right)$ of gas species $i, \varepsilon$ is the porosity, $\beta$ is the tortuosity, $Y_{i}$ is the mass fraction of species $i$ and $C_{t}$ is the thickness correction factor, see Section 4.2.1.

$D_{i j}=D_{j i}$ are binary diffusion coefficients which are calculated for each pair of gases using the expression of Fuller et al. [1] 
$D_{i j}=\frac{0.0143 T^{1.75}\left[\frac{1}{M_{i}}+\frac{1}{M_{j}}\right]^{1 / 2}}{\sqrt{2} p\left[V_{i}^{1 / 3}+V_{j}^{1 / 3}\right]}\left(\mathrm{ms}^{-2}\right)$

$D_{k, i}, A^{0}$ and $A^{\infty}$ are given by,

$$
D_{k, i}=\frac{d_{p}}{3} \sqrt{\frac{8 R T}{\pi M_{i}}}, A^{0}=\frac{32 \mu_{g}}{\rho_{g} d_{p}^{2} \sum_{i=1}^{N} Y_{i} / \sqrt{M_{i}}}, \quad A^{\infty}=\frac{3}{d_{p}}\left(\frac{R T \pi}{8}\right)^{\frac{1}{2}}
$$

These equations are re-arranged into a format suitable for CFD applications, i.e. an equation in terms of the diffusion fluxes $\mathbf{G}_{j}^{d}$

$\nabla Y_{i} \rho_{g} \frac{\varepsilon}{\beta^{2}} \frac{C_{d}}{C_{t}}=\sum_{j=1, j \neq i}^{N}\left(\frac{\mathbf{G}_{j}^{d} Y_{i}}{D_{j i}^{e}}-\frac{\mathbf{G}_{i}^{d} Y_{j}}{D_{i j}^{e}}\right)-\frac{Y_{i}\left(\varepsilon \nabla p / A \beta^{2}+\sum_{k=1}^{N} \mathbf{G}_{k}^{d} / \sqrt{M_{k}}\right)}{\sum_{k=1}^{N} Y_{k} / \sqrt{M_{k}}} \sum_{j=1}^{N}\left(\frac{Y_{j}}{D_{j i}^{e}}-\frac{Y_{j}}{D_{i j}^{e}}\right)$

and an equation for the convective mixture flux $\mathbf{G}$

$\mathbf{G}=\left(-\frac{1}{A} \nabla p \frac{\varepsilon}{\beta^{2}}-\sum_{j=1}^{N} \frac{\mathbf{G}_{j}^{d}}{\sqrt{M_{j}}}\right) / \sum_{j=1}^{N} \frac{Y_{j}}{\sqrt{M_{j}}}$

The CPIM equations can be rearranged into a system of equations based on the diffusion flux of each gas species $\mathbf{G}_{i}^{d}$ and is represented in matrix form by

$$
\begin{aligned}
& \nabla \mathbf{Y}_{(N \times 1)} \rho_{g} \frac{\varepsilon}{\beta^{2}} \frac{C_{d}}{C_{t}}=\mathbf{B}_{(N \times N)} \mathbf{G}_{(N \times 1)}^{d} \\
& B_{i i}=-\sum_{j=1, j \neq i}^{N}\left(\frac{Y_{j}}{D_{i j}^{e}}\right)-\frac{Y_{i} / \sqrt{M_{i}}}{\sum_{k=1}^{N} Y_{k} / \sqrt{M_{k}}} \sum_{j=1}^{N}\left(\frac{Y_{j}}{D_{j i}^{e}}-\frac{Y_{j}}{D_{i j}^{e}}\right) \\
& B_{i j}=\frac{Y_{i}}{D_{j i}^{e}}-\frac{Y_{i} / \sqrt{M_{j}}}{\sum_{k=1}^{N} Y_{k} / \sqrt{M_{k}}} \sum_{j=1}^{N}\left(\frac{Y_{j}}{D_{j i}^{e}}-\frac{Y_{j}}{D_{i j}^{e}}\right)
\end{aligned}
$$

These equations must be solved for use in CFD codes such as STAR-CCM+ which require a single diffusivity $D_{i}$ for each gas species to relate the species diffusion flux to its mass fraction gradient 
$\mathbf{G}_{i}^{d}=-\rho_{g} D_{i} \nabla Y_{i}$

The MPC solves the CPIM equations using an iterative procedure which yields an accurate solution to this system of equations without the requirement to invert the matrix $\mathbf{B}$.

\subsection{THERMAL MODELS}

The thermal models are used to enhance the fluid flow models by accounting for or neglecting heat transfer within the computational domain.

\subsubsection{LOW FIDELITY}

The low fidelity thermal model establishes isothermal simulations by freezing the energy solver in STAR$\mathrm{CCM}+$ to prevent any temperature changes.

\subsubsection{HIGH FIDELITY}

The high fidelity thermal model utilizes default STAR-CCM+ CFD capabilities and has the following features:

- Convective and conductive heat transfer is included by default and is described by numerical fluid flow simulation incorporating the solution of an energy conservation equation.

○ Conduction considered in both fluid and solid material components.

- Additional models are selected to describe the effects of radiation.

- Surface-to-surface model describes radiative heat transfer in a non-participating medium.

○ Discrete Ordinates Model describes radiative heat transfer in a participating medium.

\subsection{CHEMISTY MODELS}

The chemistry models are used to enhance the fluid flow models by incorporating chemical reactions into a simulation using different levels of calculation complexity.

\subsubsection{LOW FIDELITY}

The low fidelity chemistry model extends the existing default STAR-CCM+ chemistry model and has the following features:

- STAR-CCM+ "User Reaction" model and "Operator splitting" complex chemistry method are used to model the specified chemical reactions.

- Reaction rates are calculated from Arrhenius expressions.

- Source terms are incorporated into the simulation to model the effects of species and heat generation.

The general form of the reactions that can be modeled using the STAR-CCM+ "User Reaction" model is

$\sum_{i=1}^{N r} m_{i} \mathrm{R}_{i} \longleftrightarrow \sum_{j=1}^{N p} n_{j} \mathrm{P}_{j}$ 
where $\mathrm{R}$ and $\mathrm{P}$ are the reactants and products respectively, $m$ and $n$ their stoichiometric coefficients and $N r$ and $N p$ are their total number. The rate $r_{l}$ of the forward reaction is described by the following expression:

$$
r_{1}=k_{1} \prod_{i=1}^{N r}\left[\mathrm{R}_{\mathrm{i}}\right]^{m_{i}} \quad\left(\mathrm{kmol} \mathrm{m}^{-3} \mathrm{~s}^{-1}\right)
$$

Where $\left[\mathrm{R}_{\mathrm{i}}\right]$ is the molar concentration of species $\mathrm{R}_{\mathrm{i}}\left(\mathrm{kmol} \mathrm{m}^{-3}\right)$. The rate constant $k_{l}$ is described by an Arrhenius expression

$$
k_{1}=k T^{b} \exp \left(\frac{-E_{a}}{R T}\right)\left(\mathrm{kmol}^{\left(1-\sum m_{i}\right)} \mathrm{m}^{-3^{\left(1-\sum m_{i}\right)} \mathrm{s}^{-1}}\right)
$$

Where $k$ is a pre-exponential constant with the same units as $k_{1}, b$ is a dimensionless constant and $E_{a}$ is the activation energy $\left(\mathrm{J} \mathrm{kmol}^{-1}\right)$. If a reversible reaction is specified, the reverse rate constant $k_{-l}$ is determined by STAR-CCM+ using thermodynamic relations.

The low fidelity chemistry model enhances the "User Reaction" model outlined above by replacing the pre-exponential constant $k$ with a function that allows reaction rates to be set locally or switched off in specific model regions.

\subsubsection{HIGH FIDELITY}

The high fidelity chemistry model extends the advanced DARS-CFD chemistry modeling capabilities available within STAR-CCM+ and has the following features:

- Homogeneous (gas phase only) and heterogeneous (gas and surface phases) chemistry mechanisms can be modeled.

- Mechanisms can be specified in the widely used CHEMKIN format.

- Reaction rates are calculated from Arrhenius expressions using the "Operator splitting" complex chemistry method.

- Source terms are incorporated into the simulation to model the effects of species and heat generation.

- Additional information is provided about reacting surfaces including the site coverage fractions and production rates of adsorbed species.

The general form of the gas phase reactions that can be modeled using DARS-CFD is identical to that provided for the "User Reaction" model, see Section 4.4.1.

The general form of the surface phase reactions that can be modeled using DARS-CFD is,

$\sum_{i=1}^{N r} m_{i} \mathrm{R}_{i} \longleftrightarrow \sum_{j=1}^{N p} n_{j} \mathrm{P}_{j}$

Where $\mathrm{R}$ and $\mathrm{P}$ are the reactants and products respectively, $m$ and $n$ their stoichiometric coefficients and $N r$ and $N p$ are their total number. Surface adsorbed species (and empty sites), gas phase species and bulk 
solid species can participate in these reactions. The rate $r_{l}$ of the forward reaction is described by the following expression:

$$
r_{1}=k_{1} \prod_{i=1}^{N r}\left[\mathrm{R}_{\mathrm{i}}\right]^{m_{i}} \quad\left(\mathrm{~mol} \mathrm{~cm}{ }^{-2} \mathrm{~s}^{-1}\right)
$$

Where $\left[\mathrm{R}_{\mathrm{i}}\right]$ is the molar concentration of species $\mathrm{R}_{\mathrm{i}}(\mathrm{mol}, \mathrm{cm})$. Gas phase concentrations are determined from the ideal gas equation, surface concentrations are determined from the site density $\Gamma\left(\mathrm{mol} \mathrm{cm}^{-2}\right)$ and the species site fraction $\theta$ and bulk solid concentrations are set to unity.

In general the rate constant $k_{l}$ is described by an Arrhenius expression

$$
k_{1}=k T^{b} \exp \left(\frac{-E_{a}}{R T}\right) \quad\left(\mathrm{mol} \mathrm{cm} \mathrm{s}{ }^{-1}\right)
$$

where $k$ is a constant with the same units as $k_{l}, b$ is a dimensionless constant and $E_{a}$ is the activation energy $\left(\mathrm{Jmol}^{-1}\right)$.

The rate constant can also be calculated by other means including the sticking coefficient or by including an additional surface coverage dependent activation energy.

\subsection{CURRENT FLOW MODELS}

Current flow models are used to enhance the fluid flow models by including the transport of charged species (electronic and ionic) within conducting model components.

\subsubsection{LOW FIDELITY}

The low fidelity version of this model uses an analytical current flow model and has the following features:

- Model considers three cell layers, an electrode on either side of an electrolyte.

- One way coupling to low fidelity electrochemistry model:

- This model provides a local estimate of cell ohmic Area Specific Resistance (ASR) to the low fidelity electrochemistry model, see Section 4.6.1.

An analytical solution exists for the current flow in a three layer (electrode/electrolyte/electrode) cell, see Figure 5. 


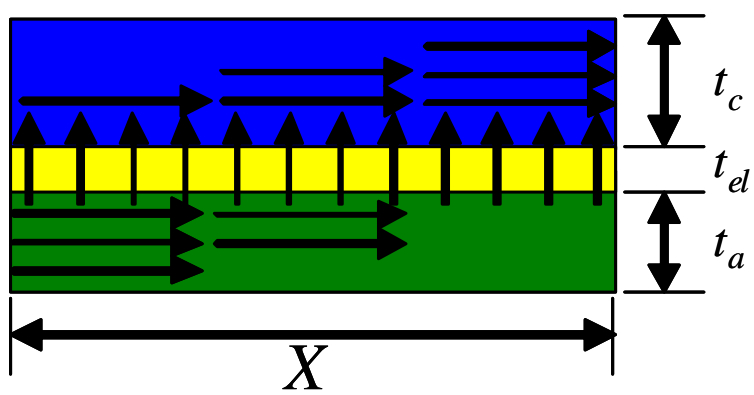

Figure 5 Three layer model for analytical current flow solution.

This analytical model is subject to the following assumptions:

- Current flow is horizontal in electrodes and vertical in electrolyte.

- Potential difference across electrode/electrolyte interfaces is neglected

- Conductivity of each layer is uniform.

The total area specific resistance $R$ of the above arrangements of cell components is given by

$R=C J[\operatorname{coth}(J)+B(J-2 \tanh (0.5 J))]$

where

$C=\frac{t_{c}}{\sigma_{c}}+\frac{t_{e l}}{\sigma_{e l}}+\frac{t_{a}}{\sigma_{a}}$

$E=\frac{t_{c} \sigma_{c}}{t_{a} \sigma_{a}}$

$B=\frac{E}{(1+E)^{2}}$

$L=\sqrt{\frac{t_{e l} / \sigma_{e l}}{\frac{1}{t_{a} \sigma_{a}}+\frac{1}{t_{c} \sigma_{c}}}}$

$J=\frac{X}{L}$

and $t_{a}, t_{e l}, t_{c}$ are the thicknesses $(\mathrm{m})$ of the three cell layers, $\sigma_{a}, \sigma_{e l}, \sigma_{c}$ are their conductivities $\left(\mathrm{Sm}^{-1}\right)$ and $X$ is the width of the cell (m).

The calculated value of $R$ is used to provide an estimate of $A S R_{O h m}$ to the low fidelity electrochemistry model, see Section 4.6.1. 


\subsubsection{HIGH FIDELITY}

The high fidelity current flow model uses a numerical current flow simulation and has the following features:

- Current flow modeled by activating the "electrodynamic electric potential" solver in STAR$\mathrm{CCM}+$.

○ Component specific conductivity supplied by user.

- Fully coupled to high fidelity electrochemistry model:

- Electrochemical reaction rates from electrochemistry model used to calculate current source terms in this model.

- This model supplies electric potential at electrode/electrolyte interfaces to the electrochemistry model.

The flow of conventional electronic current is modeled using the solution of an additional equation in STAR-CCM+ to model the differential form of Ohm's law,

$i=-\sigma \nabla \phi$

where $i$ is the local current density, $\sigma$ is the material conductivity and $\phi$ is the electric field strength. Boundary conditions are applied to this equation to represent the effects of electrochemical reactions and external connections.

\subsection{ELECTROCHEMISTRY MODELS}

The electrochemistry models are used to model the effects of electrochemical charge transfer reactions at the interface between model components.

\subsubsection{LOW FIDELITY}

The low fidelity electrochemistry model extends the chemistry modeling capabilities of STAR-CCM+. This model utilizes a pair of electrochemical reactions specified on either side of the same electrolyte, see Figure 6. 


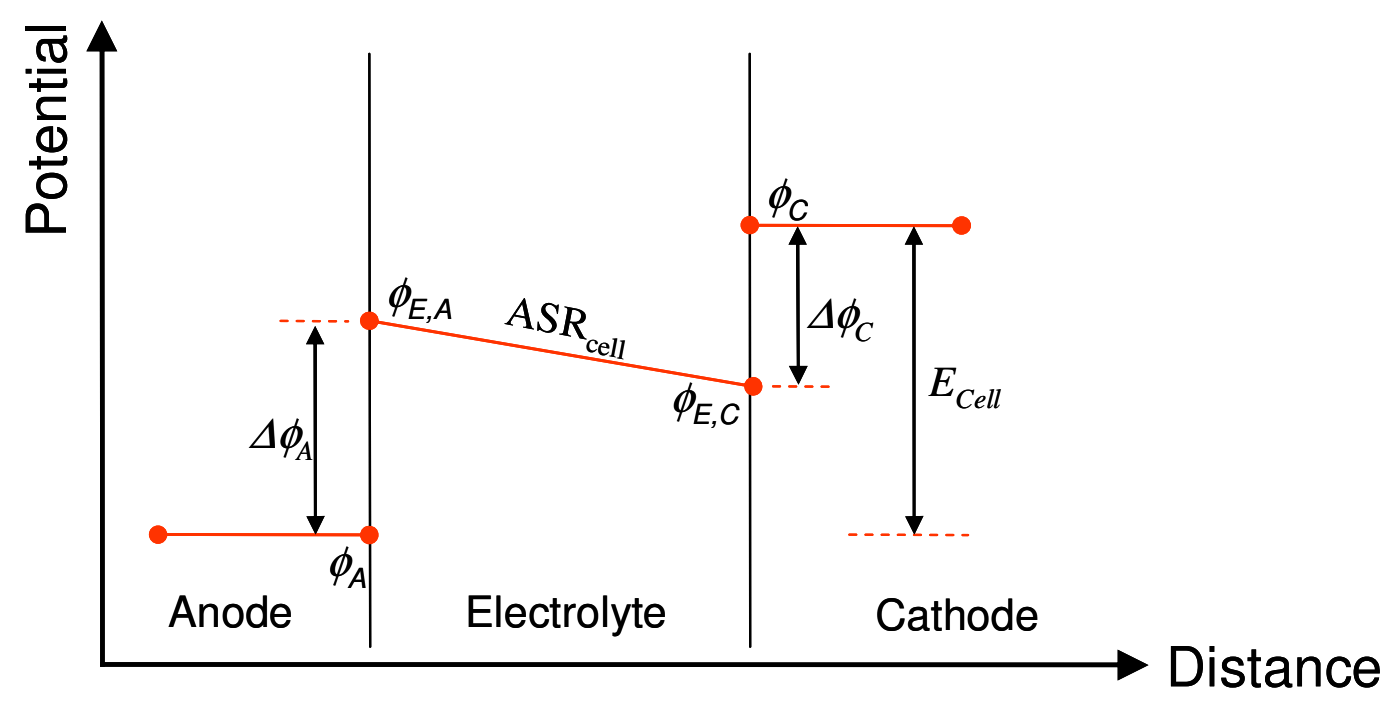

Figure 6 Variation of potential through cell layers.

The low fidelity electrochemistry model has the following features:

- Calculation result is the local value of electrode potential difference $\phi_{C^{-}} \phi_{A^{-}}$.

- Any pair of electrochemical reactions can be modeled.

- Reactions are set at each electrode/electrolyte interface.

- Source terms account for species and heat generation at interfaces.

- A uniform current density is prescribed.

- The Butler-Volmer equation is used to calculate activation losses which are incorporated into Area Specific Resistance (ASR) of the cell $A S R_{\text {cell }}$.

- An additional $A S R_{\text {cell }}$ contribution can be specified to account for ohmic losses or additional ohmic losses calculation methods can be chosen, see Section 4.5.1

- Model interfaces with either low or high fidelity chemistry models.

The general form of the reactions that can be modeled is

$$
\sum_{i=1}^{N r} m_{i} \mathrm{R}_{i} \longleftrightarrow \sum_{j=1}^{N p} n_{j} \mathrm{P}_{j}+z e^{-}
$$

where reactants and products can be either participating gas species, surface species or bulk solid species, such as $\mathrm{O}^{2-}$ ions. The equilibrium Nernst potential for a reaction is calculated using

$$
E_{N}=-\frac{\Delta G}{z F}=-\frac{\Delta G^{\circ}}{z F}+\frac{R T}{z F} \ln \left(\frac{\prod a\left[\mathrm{R}_{i}\right]^{m}}{\prod a\left[\mathrm{P}_{i}\right]^{n}}\right)
$$


Where $a\left[\mathrm{R}_{i}\right]$ is the activity of reactant $\mathrm{R}_{i}$ and $F$ is Faraday's constant. The Gibbs function for the reaction including the effects of species activity $\Delta G$ is calculated from

$\Delta G=-\sum n_{j} G_{P, j}-\sum m_{i} G_{R, i}$

Where $G$ is the Gibbs function for a reactant or product and is given by

$G_{i}=H_{i}-T S_{i}+R T \ln A_{i}$

Where the activity $A_{i}$ of species $i$ is given by $p_{i} / p^{\circ}$ for gaseous species, the surface coverage fraction $\theta_{i}$ for surface species and unity for bulk solid state species.

The molar enthalpy $\mathrm{H}_{\mathrm{i}}$ and entropy $\mathrm{S}_{\mathrm{i}}$ are determined from the 7 NASA polynomial coefficients stored in the internal STAR-CCM+ materials database, see Section 4.1.2.

The equilibrium potential across an interface $\Delta \phi$ is given by the Nernst potential $E_{N}$ and the deviation from equilibrium is accounted for by incorporating the activation loss $\Delta E_{A}$ into the cell area specific resistance $A S R_{\text {cell }}$ using,

$$
\begin{aligned}
A S R_{\text {cell }} & =A S R_{\text {Ohm }}+A S R_{\text {ActA }}+A S R_{\text {ActC }} \\
A S R_{\text {Act }} & =\frac{\Delta E_{A}}{i}
\end{aligned}
$$

where $A S R_{A c t A}$ and $A S R_{A c t C}$ are the anode and cathode activation ASR and $i$ is the prescribed uniform local current density.

$\Delta E_{A}$ is calculated using the Butler-Volmer equation

$$
i=i_{0}\left[\exp \left(\frac{\alpha z F \Delta E_{A}}{R T}\right)-\exp \left(\frac{-(1-\alpha) z F \Delta E_{A}}{R T}\right)\right]\left(\mathrm{Am}^{-2}\right)
$$

where $i$ and $i_{0}$ are the current density and the exchange current density respectively and $\alpha$ the symmetry factor. It is assumed that $\alpha=0.5$ and the above equation is solved to find $\Delta E_{A}$

$$
\Delta E_{A}=\left(\frac{R T}{z F}\right) \sinh ^{-1}\left(\frac{i}{2 i_{0}}\right)
$$

The exchange current density $i_{0}$ is calculated using the following expression,

$$
i_{0}=k \exp \left(\frac{-E_{a}}{R T}\right) \prod_{i=1}^{N r} a\left[\mathrm{R}_{i}\right]^{u_{i}} \prod_{j=1}^{N p} a\left[\mathrm{P}_{j}\right]^{v_{j}}\left(\mathrm{Am}^{-2}\right)
$$


where $k$ is a pre-exponential factor $\left(\mathrm{Am}^{-2}\right), E_{a}$ is the activation energy $\left(\mathrm{Jmol}^{-1}\right), u_{i}$ and $v_{i}$ are user defined reaction orders.

The calculated cell potential $\phi_{C^{-}} \phi_{A}$.is found from

$\phi_{C}-\phi_{A}=E_{N_{A}}+E_{N C}-\frac{A S R_{c e l l}}{i}$

Flux terms for the consumption of participating species are applied as boundary conditions to each interface using the following equation.

$$
S_{r}=\frac{i M_{r}}{z F}\left(\mathrm{kgm}^{-2} \mathrm{~s}^{-1}\right)
$$

where $M_{r}$ is the molar mass of species $r$. The applied species flux boundary conditions automatically release an amount of heat equivalent to the enthalpy of the electrochemical reaction and it is therefore necessary to recover the heat that is converted into electrical work. The corresponding heat flux term applied to the interface is

$$
S_{H}=-i \Delta \phi\left(\mathrm{Jm}^{-2} \mathrm{~s}^{-1}\right)
$$

\subsubsection{HIGH FIDELITY}

The high fidelity electrochemistry model utilizes the same approach outlined for the low fidelity electrochemistry model in Section 4.6.1. Additional features are provided for this model to facilitate direct coupling with the high fidelity current flow model:

- Cell potential is calculated using potential field data from the high fidelity current flow simulation.

- Electrochemical reaction rate is calculated using the cell potential and flux terms are returned for participating gases and charged species.

Further features are also provided to allow the stable calculation of fuel cell performance under fuel starvation conditions. These features are as follows:

- Multiple reactions can be defined on an electrode/electrolyte interface.

- User specified weighted average used to determine proportion of total current density that applies to each reaction.

- User specified weighted average used to calculate the Nernst potential of several reactions.

The general form of the high fidelity electrochemistry model is identical to that described in Section 4.6.1 for the low fidelity model. The local current density $i$ is found from the potential difference $\Delta \phi_{E}$ across the electrolyte,

$$
i=\frac{\Delta \phi_{E}}{A S R_{\text {cell }}}
$$


Where $\Delta \phi_{E}$ is calculated from $\Delta \phi_{E}=\left(\phi_{C}-E_{N C}\right)-\left(\phi_{A}+E_{N A}\right)$ and $\phi_{C}$ and $\phi_{A}$ are provided by the high fidelity current flow model.

$A S R_{\text {cell }}$ is adjusted to only take account of the activation losses and the ionic resistance of the electrolyte,

$$
\begin{aligned}
& A S R_{\text {cell }}=A S R_{\text {Ionic }}+A S R_{\text {ActA }}+A S R_{\text {ActC }} \\
& A S R_{\text {Ionic }}=\frac{t_{e}}{\sigma_{e}}
\end{aligned}
$$

where $t_{e}$ is the electrolyte thickness.

The thermodynamic and kinetic calculations have been improved from the low fidelity model to account for multiple electrochemical reactions at each interface. A Nernst potential $E_{N}$

$$
E_{N}=-\frac{\Delta G}{z F}=-\frac{\Delta G^{\circ}}{z F}+\frac{R T}{z F} \ln \left(\frac{\prod a\left[\mathrm{R}_{i}\right]^{n}}{\prod a\left[\mathrm{P}_{i}\right]^{n}}\right)
$$

and exchange current density $i_{0}$ are calculated for each reaction

$$
i_{0}=k X \exp \left(\frac{-E_{a}}{R T}\right) \prod_{i=1}^{N r} a\left[\mathrm{R}_{i}\right] \prod_{j=1}^{u_{i}} a\left[\mathrm{P}_{j}\right]^{v_{j}}\left(\mathrm{Am}^{-2}\right)
$$

A user defined ratio is used to determine a weighted average of $E_{N}$ for the reactions being considered for use by the MPC electrochemistry solution procedure. The same averaging process is also applied to the exchange current densities for each reaction. A second averaging term is then used to assign a proportion of the calculated current density to each of the electrochemical reactions.

In this way it is possible to define a set of reactions (one for fuel rich conditions and one for fuel starved conditions) that can be interpolated between to describe cell performance during the onset of fuel starvation.

\subsection{DEGRADATION MODELS}

The degradation models are used to account for the time dependent changes in material properties that occur in SOFCs after long periods of operation.

\subsubsection{LOW FIDELITY}

This model is used to describe time dependent cell properties using a simple reaction rate calculation that can be adjusted empirically to match observed cell degradation behavior. It has the following features:

- The model can be applied to a wide range of existing model properties that are installed using the MPC user interface. 
- The model assumes each time dependent property undergoes a reaction that proceeds from an initial state to an equilibrium state after an infinite amount of time has passed or until a specified reaction rate limit has been reached.

- The rate of the reaction is calculated using a specified pre-exponential term and an activation energy term.

- A specified amount of elapsed time is used to update the time dependent variables at the start of a steady-state simulation.

- Degradation conditions such as temperature can be specified independently of the simulation conditions.

It has been observed from short term cell durability tests ( 4000 hours) that changes in cell component properties over time follow a trend that can be described as a chemical reaction that proceeds toward a long term equilibrium value where the reaction rate is proportional to how far the reaction is from this equilibrium value. The reaction rate $r(t)$ at a time $t$ is given by,

$$
r(t)=k\left(1-\frac{f(t)}{f(\infty)}\right)
$$

where $f(t)$ is the value of the time dependent variable representing a cell component property at a time $t$ and $f(\infty)$ is the value of this variable when the reaction is at equilibrium $(t=\infty)$. The rate coefficient $k$ is given by

$$
k=k_{1} \exp \left(\frac{-E_{a}}{R T_{d}}\right)
$$

where $k_{l}$ is a pre-exponential term and $E_{a}$ is the activation energy $\left(\mathrm{Jmol}^{-1}\right)$ and $T_{d}$ is the degradation temperature (which is specified independently of the simulation temperature).

The calculated value of $f(t)$ is found from the following equation

$$
f(t)=(f(0)-f(\infty)) \exp \left(\frac{-k t}{f(\infty)}\right)+f(\infty)
$$

where $f(0)$ is the initial value of the time dependent variable at time $t=0$. This equation is used to update the time dependent variables for each simulation. If a reaction rate limit $r_{\text {limit }}$ is specified then the calculated value of $f(t)$ proceeds on its approach to equilibrium until the reaction rate $r(t)$ reaches $r_{\text {limit }}$. The value of $f(t)$ in this limiting case is given by

$$
f(t)=f(\infty)\left(1-\frac{r_{\text {limit }}}{k}\right)
$$

This model predicts an exponential decay from an initial value to a long term equilibrium value or until a prescribed rate of reaction is reached. Although this trend may not be suitable for all degradation 
processes, several reactions can be combined together to yield more complex behavior or alternatively new models can be added to describe different trends.

The purpose of this model is to allow SOFC simulations to include time dependent properties that have been derived from empirical curve fits to experimental data. This model is not based on any underlying physical process and therefore it cannot predict cell durability beyond the test times used to obtain its empirical constants. However, it is possible to apply models derived from small scale testing to larger simulations.

\subsubsection{HIGH FIDELITY}

This model is used to describe time dependent cell properties using a kinetic reaction mechanism to calculate the rate of a degradation process. It has the following features:

- The model can be applied to a wide range of existing model properties that are installed using the GUI.

- The model uses a kinetic reaction mechanism to calculate the rate of change of a time dependent model property.

- The model can be combined with the low fidelity empirical degradation using the reaction rate limit option described in Section 4.7.1.

- The reaction mechanism is specified in the CHEMKIN format in the same manner as for high fidelity chemistry calculations, see Section 4.4.2.

The rate of reaction $r(t)$ calculated from the specified reaction mechanism is used to update $f(t)$, the value of a time dependent variable using

$f(t)=f(0)-r(t) \times \Delta t$

where $f(0)$ is the initial value of the time dependent variable at time $t=0$ and $\Delta t$ is the specified elapsed time.

Several time dependent variables can be combined together to model complex degradation processes and these variables can feedback boundary conditions to the kinetic reaction calculations.

The purpose of this model is to establish the capability to model degradation processes using physical mechanisms. The details of these mechanisms are derived from an extensive amount of experimentation and measurement which is beyond the scope of this project.

\subsection{TIME MODEL}

The time models are used to enhance the fluid flow models selecting whether a steady-state or transient analysis is required.

\subsubsection{LOW FIDELITY}

The low fidelity time model is used to simulate steady-state behavior and utilizes the STAR-CCM+ "Steady" solver, which is activated by default when in the base fluid flow models, see Section 4.1.2. 


\subsubsection{HIGH FIDELITY}

The high fidelity time model is used to simulate transient behavior and utilizes the STAR-CCM+ "Unsteady-Implicit" in place of the standard "Steady" solver. The implementation of this model depends on the size of the prescribed time step.

When time steps are large $(>0.1 \mathrm{~s})$ and it can be assumed that SOFC current flow, electrochemical and chemical reactions are able to reach a steady-state and the following steps are taken by the MPC:

- A factor is applied to chemical reaction rate coefficients to reduce their magnitude and allow STAR-CCM to calculate their steady state independently of time step size. A correction is then applied to amplify the applied reaction rates back to their original magnitude.

- MPC calculations are only updated at the start of each time step as it is not possible to stop and restart the transient iterations without compromising the time accuracy of the solution. Therefore it can be necessary to break up a large time step into several smaller steps.

When time steps are very small $(<<0.1 \mathrm{~s})$ it also necessary to model the time dependent behavior of the fuel cells and the following additional steps are taken by the MPC:

- It is no longer necessary to modify chemical reaction rates to apply steady state assumptions.

- Electrochemistry models are enhanced to include capacitance of each electrode/electrolyte interface.

In very small time step transient operation the local current density $i$ across an electrode/electrolyte interface is given by

$i=i_{T}+i_{C}$

where $i_{T}$ and $i_{C}$ are the current densities resulting from the charge transfer electrochemical reactions and the charging/discharging of the electrical double layers respectively. The latter results from voltage transients and is given by

$$
i_{C}=C \frac{\partial \phi}{\partial t}
$$

Where $C$ is the capacitance per unit area of electrochemical interface and $\phi$ is the potential difference across the interface. The calculation of $i_{C}$ is incorporated into the MPC electrochemistry solution procedure for transient calculations when the prescribed time step is of an appropriate size.

\section{MODEL VERIFICATION AND VALIDATION}

The project plan includes a model verification and validation work program that was run in parallel to the code enhancement program outlined in Section 4 and executed in stages through the eight planned code releases. This program includes some activities that validate model predictions against experimental data and other computational results and other activities that demonstrate the effective operation of new modeling features. These activities are outlined in the following subsections. 


\subsection{SINGLE CELL MODEL VALIDATION}

The cell components required for a single cell model are shown in Figure 7.

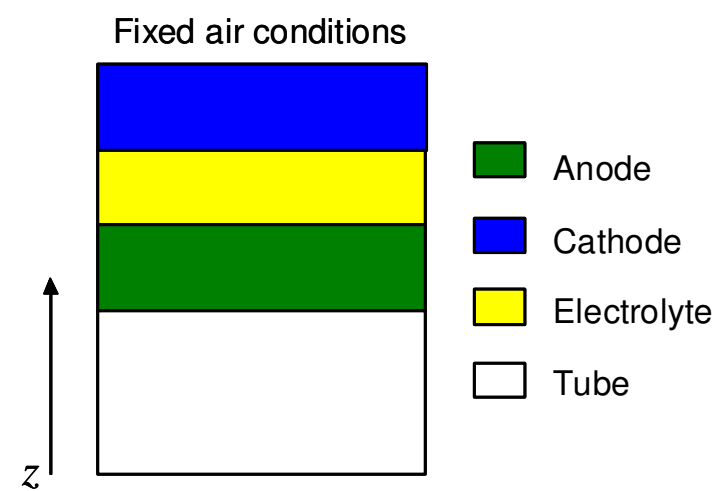

Fixed fuel conditions

Figure 7 One-dimensional model of cell components

The model configuration and computational mesh required in STAR-CCM+ to represent these components is shown in Figure 8.

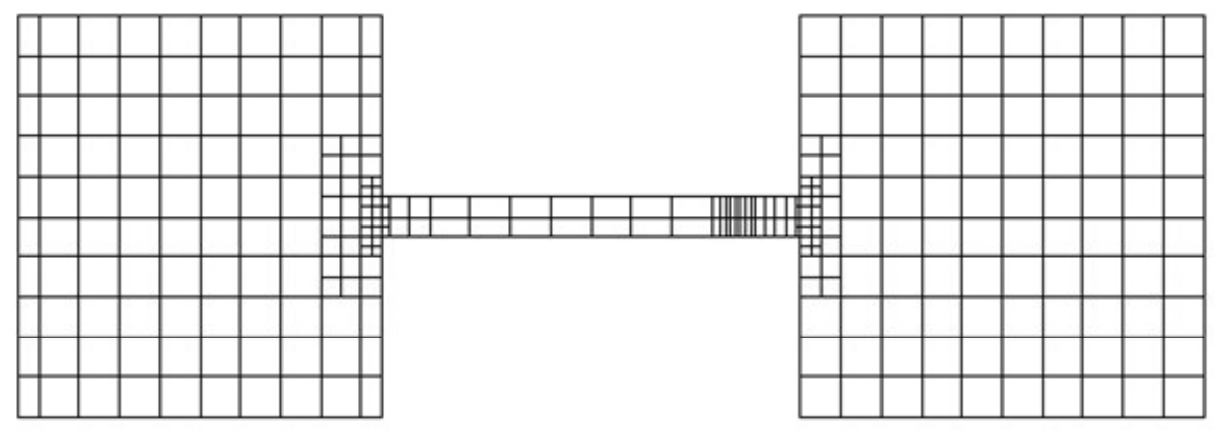

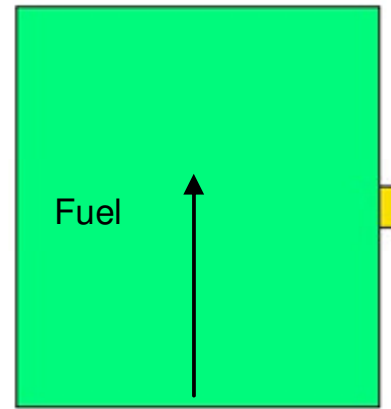

Inlet conditions

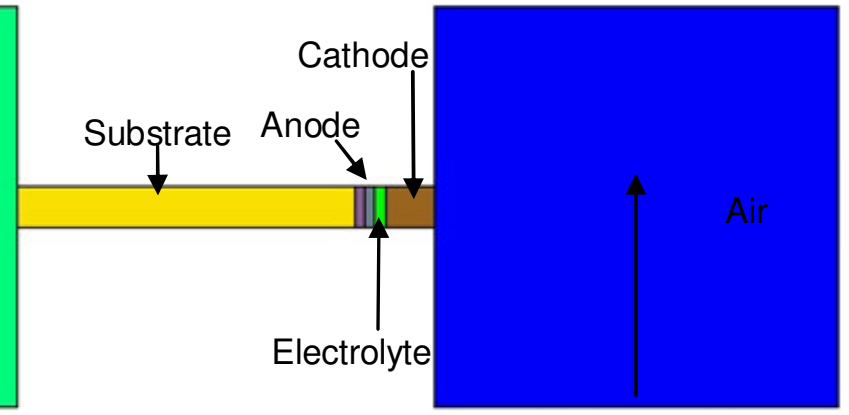

Inlet conditions

Figure 8 MPC single cell model

Figure 8 shows that additional flow channels are required in STAR-CCM+ to provide the desired fixed conditions on either side of the cell components. 
The following physical models were installed using the GUI to create a realistic single cell model:

- High fidelity fluid flow to describe the flow of gases in the porous cell components and the adjacent gas channels.

- High fidelity porous flow model to provide a complete description of mass transport in the porous cell components and enhance the diffusion calculation in the adjacent gas channels. Material properties for this model were obtained from specific tests on individual cell components.

- Low fidelity thermal model to ensure isothermal operation.

- Low fidelity chemistry model to capture the behavior of the water gas shift reaction $\mathrm{H}_{2} \mathrm{O}+\mathrm{CO} \longleftrightarrow \mathrm{CO}_{2}+\mathrm{H}_{2}$ which is catalyzed by the anode material. Kinetic constants for this reaction were obtained from previous studies [2].

- Low fidelity electrochemistry model to specify the following electrochemical reactions at the cathode and anode/electrolyte interfaces respectively:

$$
\begin{array}{ll}
\circ \mathrm{O}_{2}+2 \mathrm{~V}_{\mathrm{O}}^{\bullet \bullet}(\mathrm{YSZ})+4 \mathrm{e}^{-} \longleftrightarrow 2 \mathrm{O}_{\mathrm{O}}^{\mathrm{X}}(\mathrm{YSZ}) \\
\circ \mathrm{H}_{2}+\mathrm{O}_{\mathrm{O}}^{\mathrm{X}}(\mathrm{YSZ}) \longleftrightarrow \mathrm{H}_{2} \mathrm{O}+2 \mathrm{e}^{-}+\mathrm{V}_{\mathrm{O}}^{\bullet \bullet}(\mathrm{YSZ})
\end{array}
$$

The kinetic constants required for the electrochemistry models were determined from other validation work performed by LGFCS.

- Low fidelity time model for steady-state calculations.

This model was used to compare predicted current-voltage (IV curve) behavior against experimental measurement run using a wide range of prescribed operating conditions including: pressure, temperature, fuel composition $\left(\mathrm{H}_{2}, \mathrm{H}_{2} \mathrm{O}, \mathrm{CO}, \mathrm{CO}_{2}, \mathrm{~N}_{2}\right)$ and air composition $\left(\mathrm{N}_{2}, \mathrm{O}_{2}\right)$. In total, 31 different test cases were considered and the results from these comparisons are summarized below.

Figure 9 shows a typical plot of hydrogen mole fraction within the fuel side cell components and illustrates the change in fuel composition caused by the electrochemical consumption of hydrogen at the anode/electrolyte interface (far right of plot).
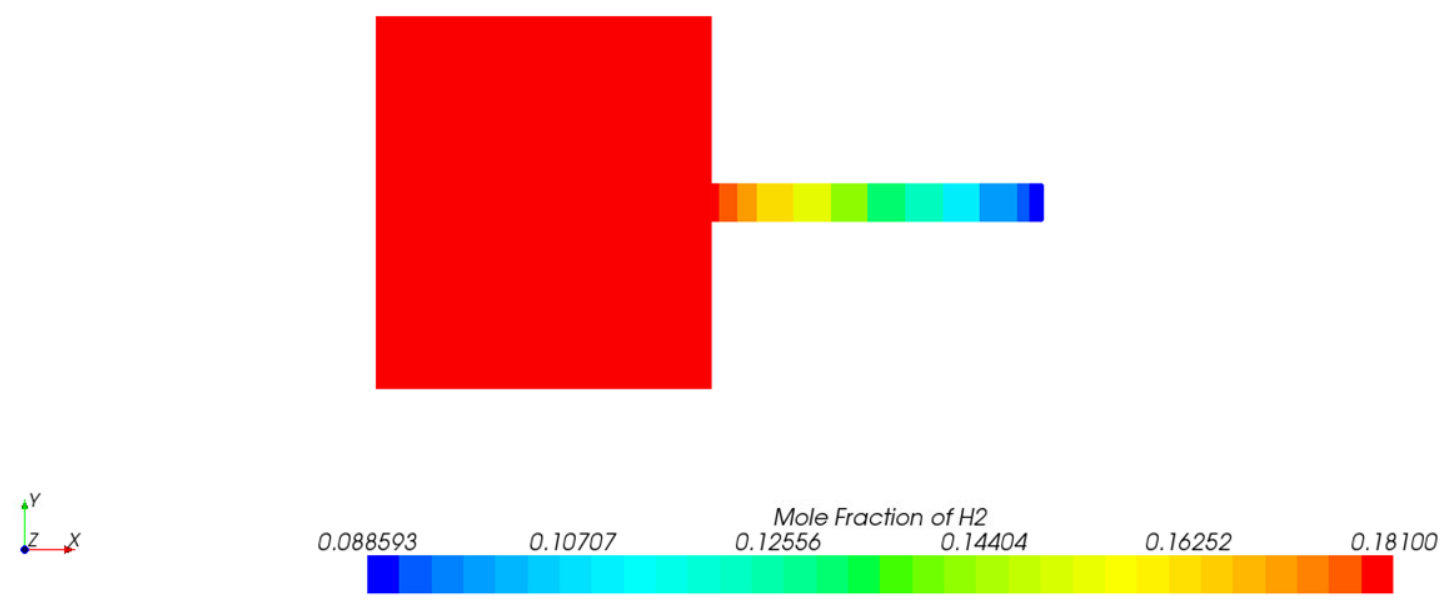

Figure 9 Hydrogen mole fraction distribution in the fuel side cell components. 
Similarly, steam is generated at the anode/electrolyte interface and there is a net mass flux away from this interface to the fuel channel. Figure $\mathbf{1 0}$ shows the variation in pressure required to support this convective flux.

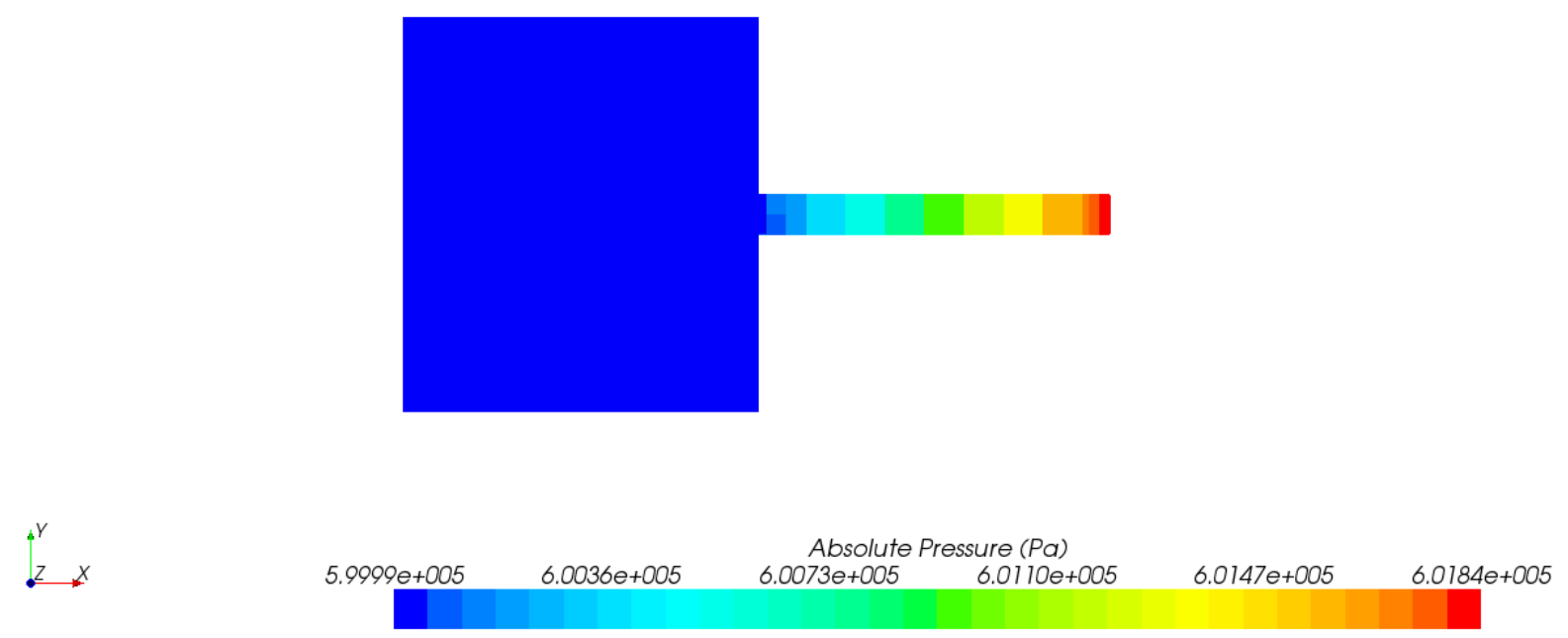

Figure 10 Pressure distribution in the fuel side cell components.

The porous flow processes illustrated above affect the cell IV characteristics. Figure 11 shows a typical predicted IV curve and a comparison to single cell experimental data. 


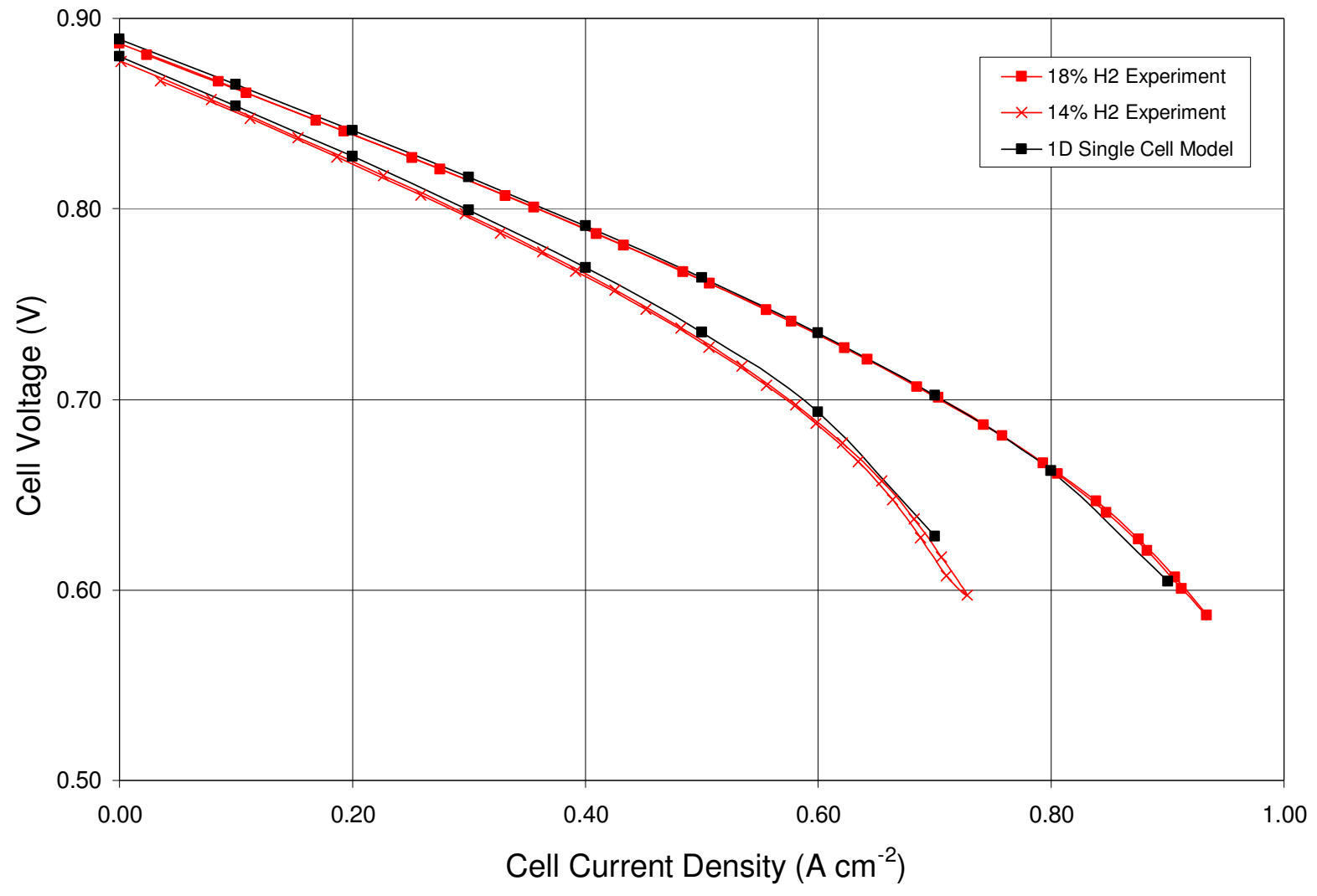

Figure 11 IV curve comparison between MPC single cell model predictions and experimental data.

Figure 11 illustrates the high the level of accuracy that is possible with the MPC modeling approach. Numerical errors were calculated by comparing experimental data and numerical predictions for three different performance measurements:

- Open circuit potential $E_{\text {cell }}(0)$

- Cell potential at maximum current density $E_{\text {cell }}\left(i_{\max }\right)$

- Cell area specific resistance $A S R$ where $A S R=\left(E_{\text {cell }}(0)-E_{\text {cell }}\left(i_{\max }\right)\right) / i_{\text {max }}$

Figure 12 shows a plot of calculated $E_{\text {cell }}(0)$ numerical errors for the 31 test cases considered and illustrates the typical level of numerical error obtained from MPC calculations. 


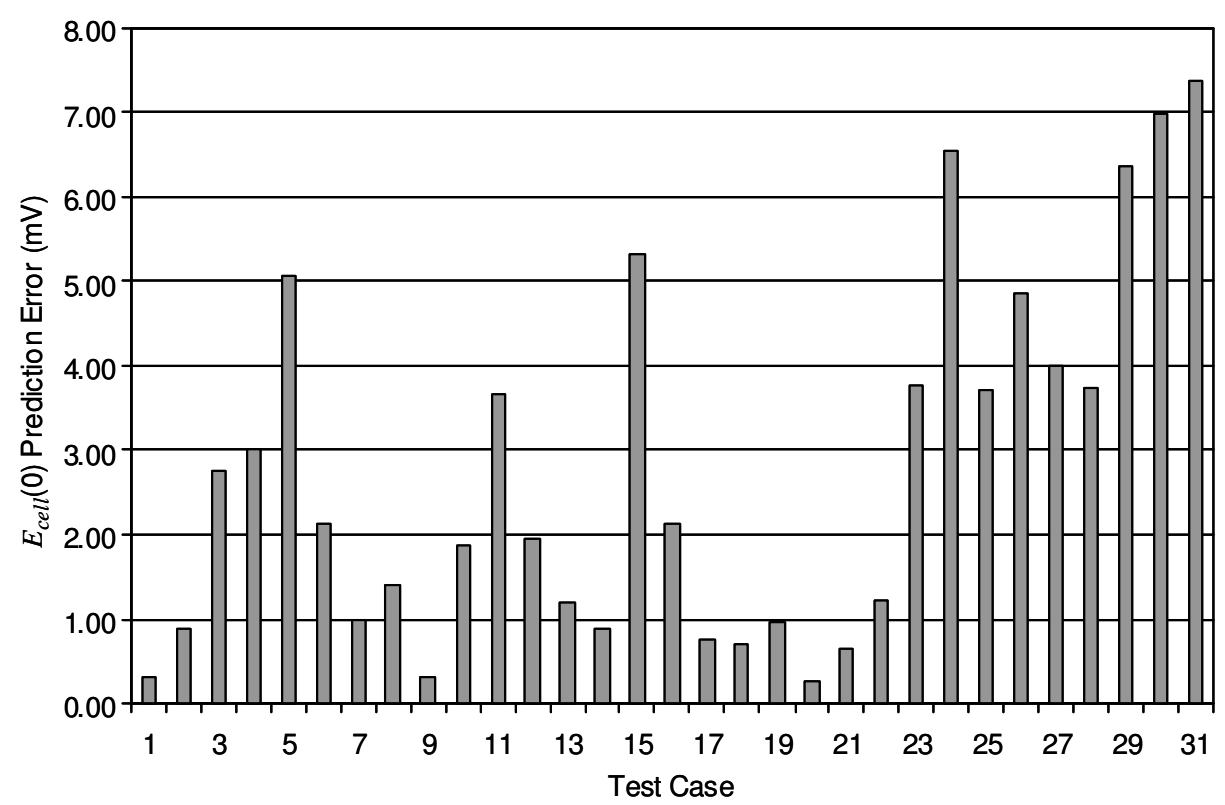

Figure 12 Calculated $E_{\text {cell }}(0)$ error magnitude for all 31 test cases.

A summary of overall numerical error is provided in Table 2 below and confirms that it was possible to validate a set of empirical model constants for LGFCS SOFC technology

Table 2 Summary of numerical errors for single cell tests.

\begin{tabular}{|l|l|l|}
\hline Numerical Prediction & Mean Error $(\%)$ & Maximum Error $(\%)$ \\
\hline$E_{\text {cell }}(0)$ & 0.3 & 0.9 \\
\hline$E_{\text {cell }}\left(i_{\max }\right)$ & 1.7 & 5.8 \\
\hline$A S R$ & 4.4 & 14.5 \\
\hline
\end{tabular}

\subsection{PENTA CELL MODEL VALIDATION}

The configuration of a two-dimensional model of the penta cell test piece shown in Figure 1(b) is shown in Figure 13. 


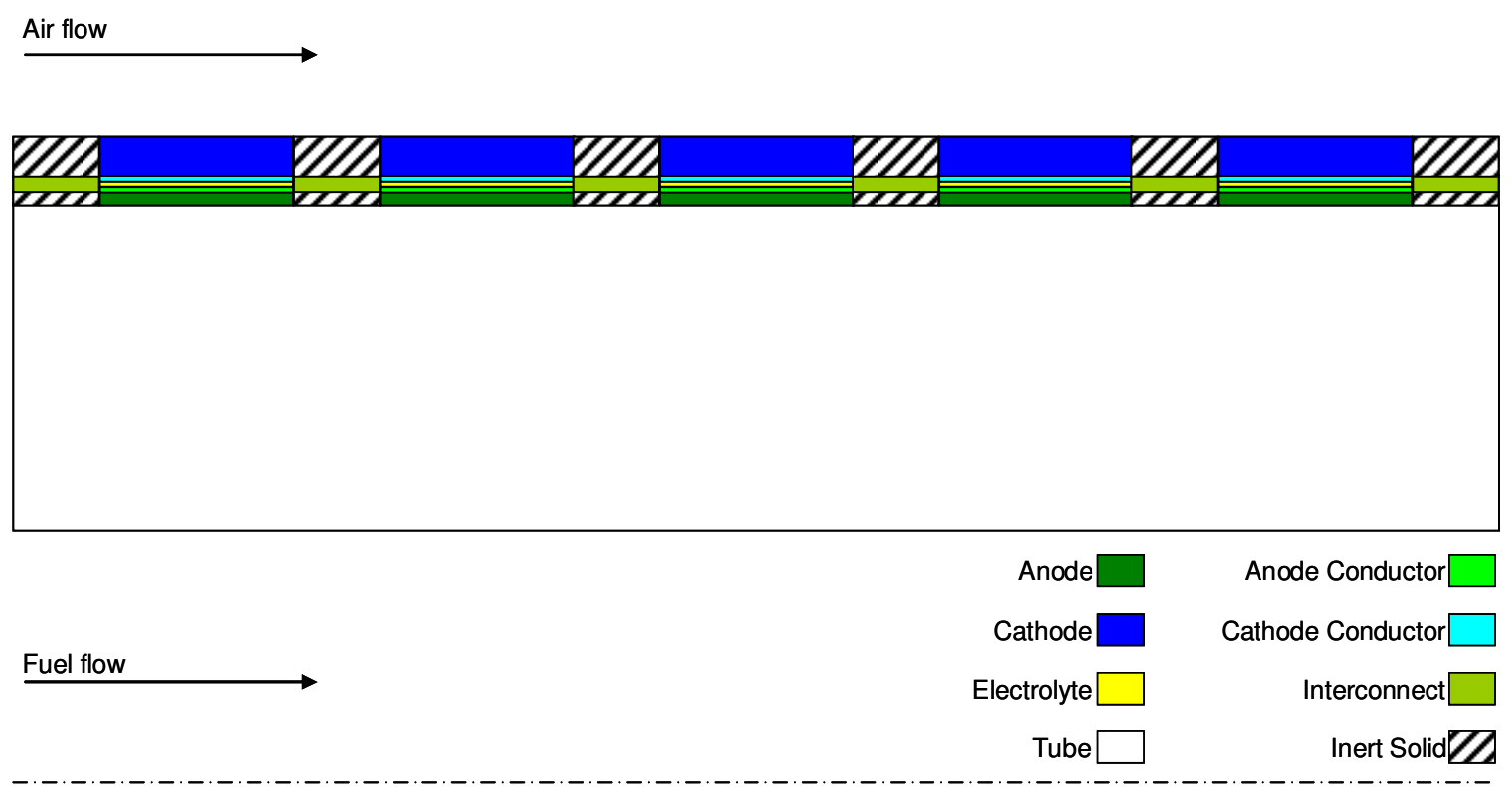

Figure 13 Two-dimensional model of penta cell test piece.

This model includes five fuel cells fabricated on the outer surface of the tube, an internal fuel channel and an external air flow channel. This model is intended for use with the high fidelity current flow model, see Section 4.5.2, and as such additional current carrying "conductor" components are specified to enable the simulation of current flow in solid model components.

Figure 14 shows a plot of a typical computational mesh required for this application and highlights the differing size and aspect ratio grid cells required to model the cell components accurately.

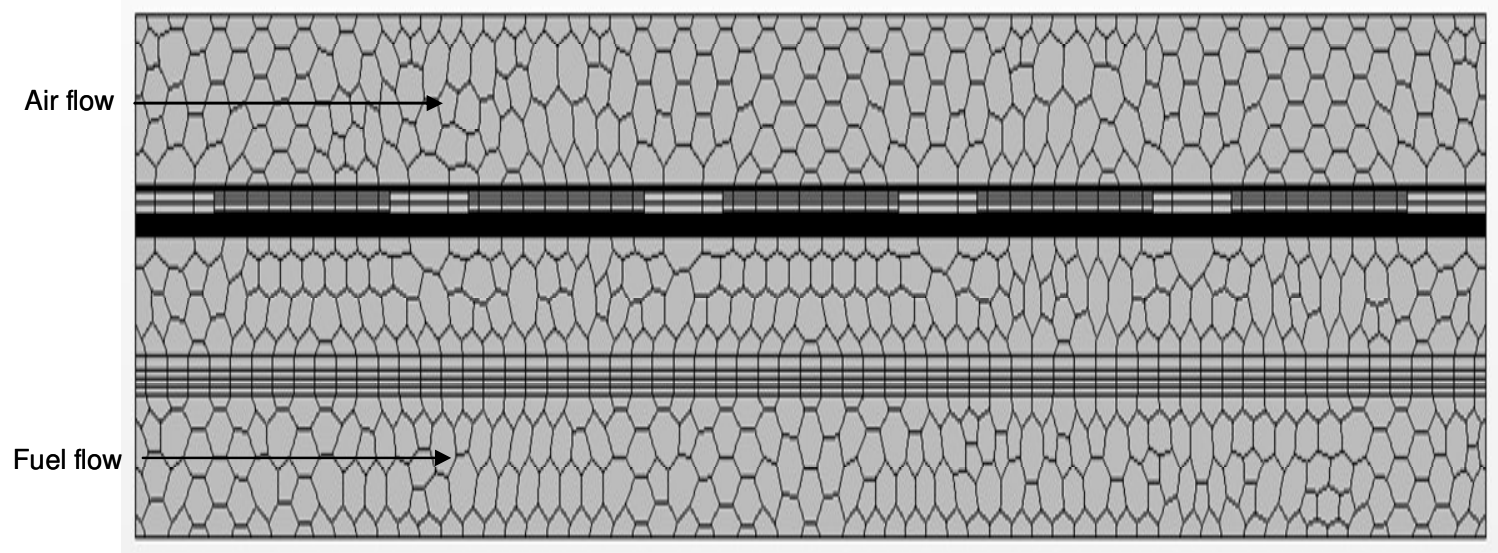

Figure 14 STAR-CCM+ two-dimensional penta cell mesh.

The following physical models were installed using the GUI to create an accurate penta cell model: 
- High fidelity fluid flow to describe the flow of gases in the porous cell components and the adjacent gas channels.

- High fidelity porous flow model to provide a complete description of mass transport in the porous cell components and enhance the diffusion calculation in the adjacent gas channels. Material properties for this model were obtained from specific tests on individual cell components.

- Low fidelity thermal model to ensure isothermal operation.

- Low fidelity chemistry model to capture the behavior of the water gas shift reaction $\mathrm{H}_{2} \mathrm{O}+\mathrm{CO} \longleftrightarrow \mathrm{CO}_{2}+\mathrm{H}_{2}$ which is catalyzed by the anode material. Kinetic constants for this reaction were obtained from previous studies [2].

- High fidelity electrochemistry model to specify the following electrochemical reactions at the cathode and anode/electrolyte interfaces respectively:

$$
\begin{array}{ll}
\circ & \mathrm{O}_{2}+2 \mathrm{~V}_{\mathrm{O}}^{\bullet \bullet}(\mathrm{YSZ})+4 \mathrm{e}^{-} \longleftrightarrow 2 \mathrm{O}_{\mathrm{O}}^{\mathrm{X}}(\mathrm{YSZ}) \\
\circ & \mathrm{H}_{2}+\mathrm{O}_{\mathrm{O}}^{\mathrm{X}}(\mathrm{YSZ}) \longleftrightarrow \mathrm{H}_{2} \mathrm{O}+2 \mathrm{e}^{-}+\mathrm{V}_{\mathrm{O}}^{\bullet \bullet}(\mathrm{YSZ})
\end{array}
$$

The kinetic constants required for the electrochemistry models were determined from other validation work performed by LGFCS.

- High fidelity current flow model to numerically simulate the flow of electric current in each conducting cell component and to fully couple the current flow simulation to the electrochemical reactions.

- Low fidelity time model for steady-state calculations.

This model was used to compare predicted current-voltage (IV curve) behavior against experimental measurement run using a wide range of prescribed operating conditions including: pressure, temperature, fuel composition $\left(\mathrm{H}_{2}, \mathrm{H}_{2} \mathrm{O}, \mathrm{CO}, \mathrm{CO}_{2}, \mathrm{~N}_{2}\right)$ and air composition $\left(\mathrm{N}_{2}, \mathrm{O}_{2}\right)$. In total, 34 different test cases were considered and the results from these comparisons are summarized below.

Figure 15 shows a typical plot of $\mathrm{H}_{2}$ mole fraction within the fuel side cell components and illustrates the change in fuel composition caused by the electrochemical consumption of $\mathrm{H}_{2}$ at the anode/electrolyte interfaces and the effect this has on $\mathrm{H}_{2}$ utilization along the length of the internal fuel channel.

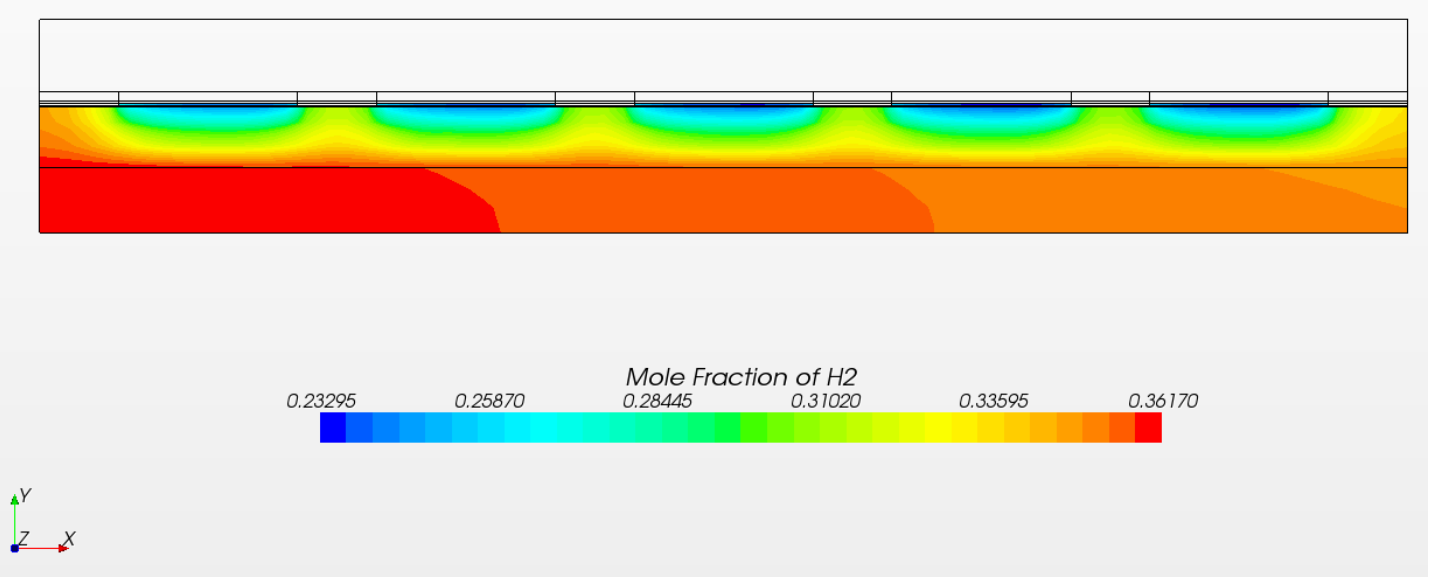

Figure 15 Hydrogen mole fraction distribution in the fuel side cell components. 
Carbon monoxide is also consumed by the fuel cells as a result of the water gas shift reaction which is catalyzed by the anode material. Figure 16 shows a plot of $\mathrm{CO}$ mole fraction distribution within the fuel side components of the model and shows that the overall $\mathrm{CO}$ utilization is less than that of $\mathrm{H}_{2}$ shown above.

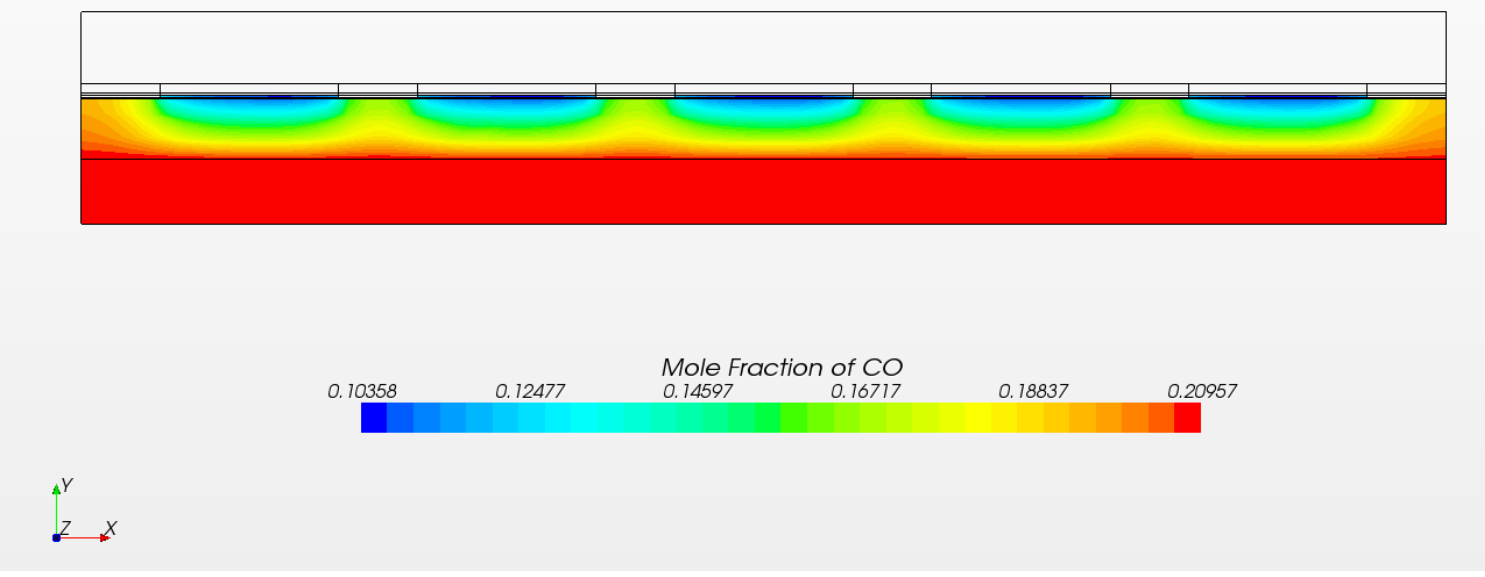

Figure 16 Carbon monoxide mole fraction distribution in the fuel side model components.

The coupled simulation of current flow and electrochemistry using their high fidelity models provides a means to investigate the effects of cell design on the current density distribution within each fuel cell electrolyte, see Figure 17.

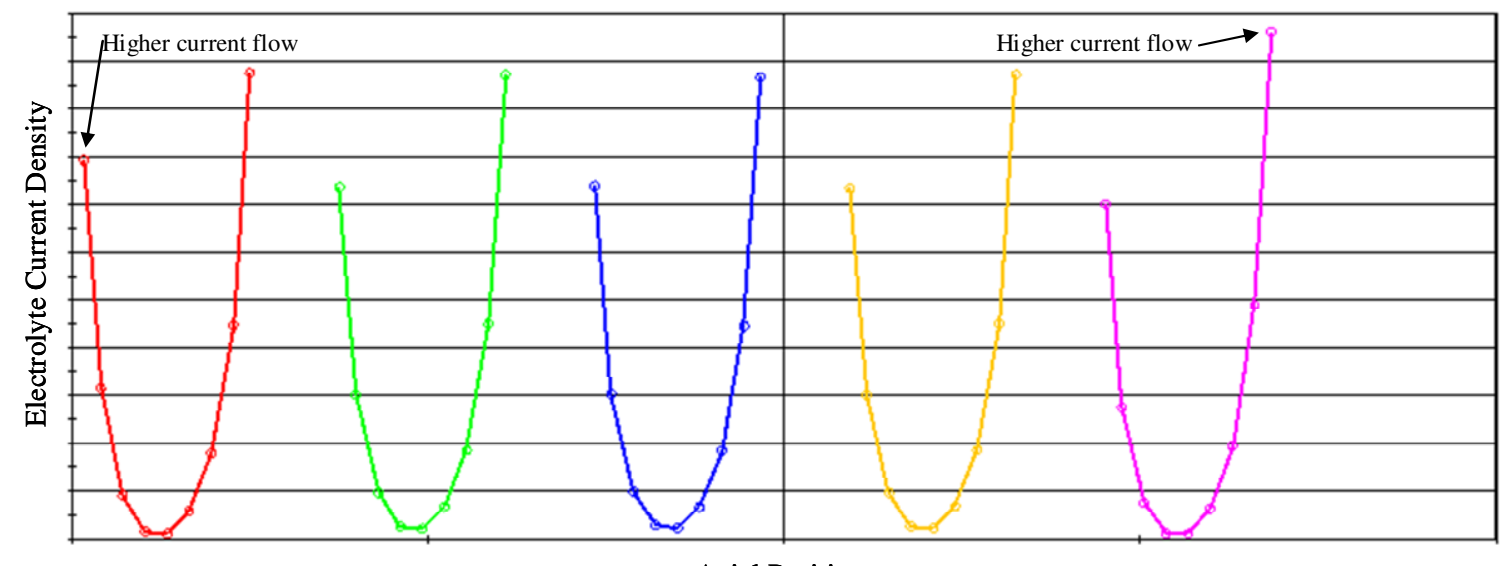

Axial Position

Figure 17 Current density distribution in each electrolyte of the penta cell model.

Figure 17 confirms that the fuel cells behave as expected with higher current densities near the edges of each electrolyte [4]. However, the current density distribution is not identical in all five cells and there is a tendency to promote current flow near the outermost edge of the first and last cells. This behavior results from the improved gas flow in these regions as the first and last cells have no upstream or downstream neighbors respectively. 
The calculated results shown in Figure 17 are directly influenced by the coupling between fuel cell processes as described by the choice of models. This coupling also controls the cell IV characteristics and Figure 18 shows a comparison between predicted values and experimental measurements.

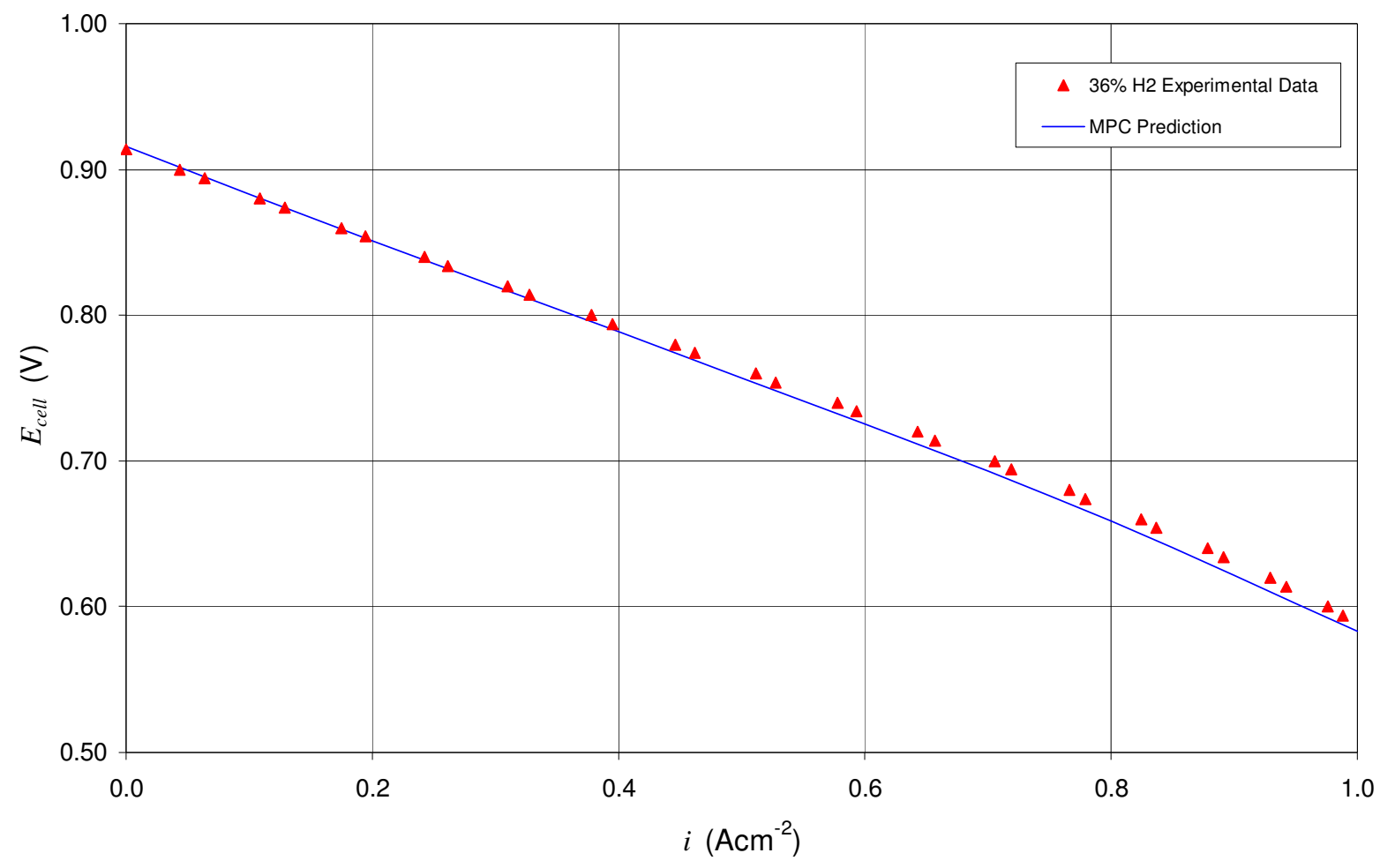

Figure 18 IV curve comparison between MPC penta cell model predictions and experimental data.

Figure 18 illustrates the high the level of accuracy that is possible with the MPC modeling approach. Numerical errors were calculated by comparing experimental data and numerical predictions for three different performance measurements: $E_{\text {cell }}(0), E_{\text {cell }}\left(i_{\max }\right)$ and $A S R$, see Section 5.1. Figure 19 shows a plot of calculated $E_{\text {cell }}(0)$ numerical errors for the 20 test cases considered and illustrates the typical level of numerical error obtained from MPC calculations. 


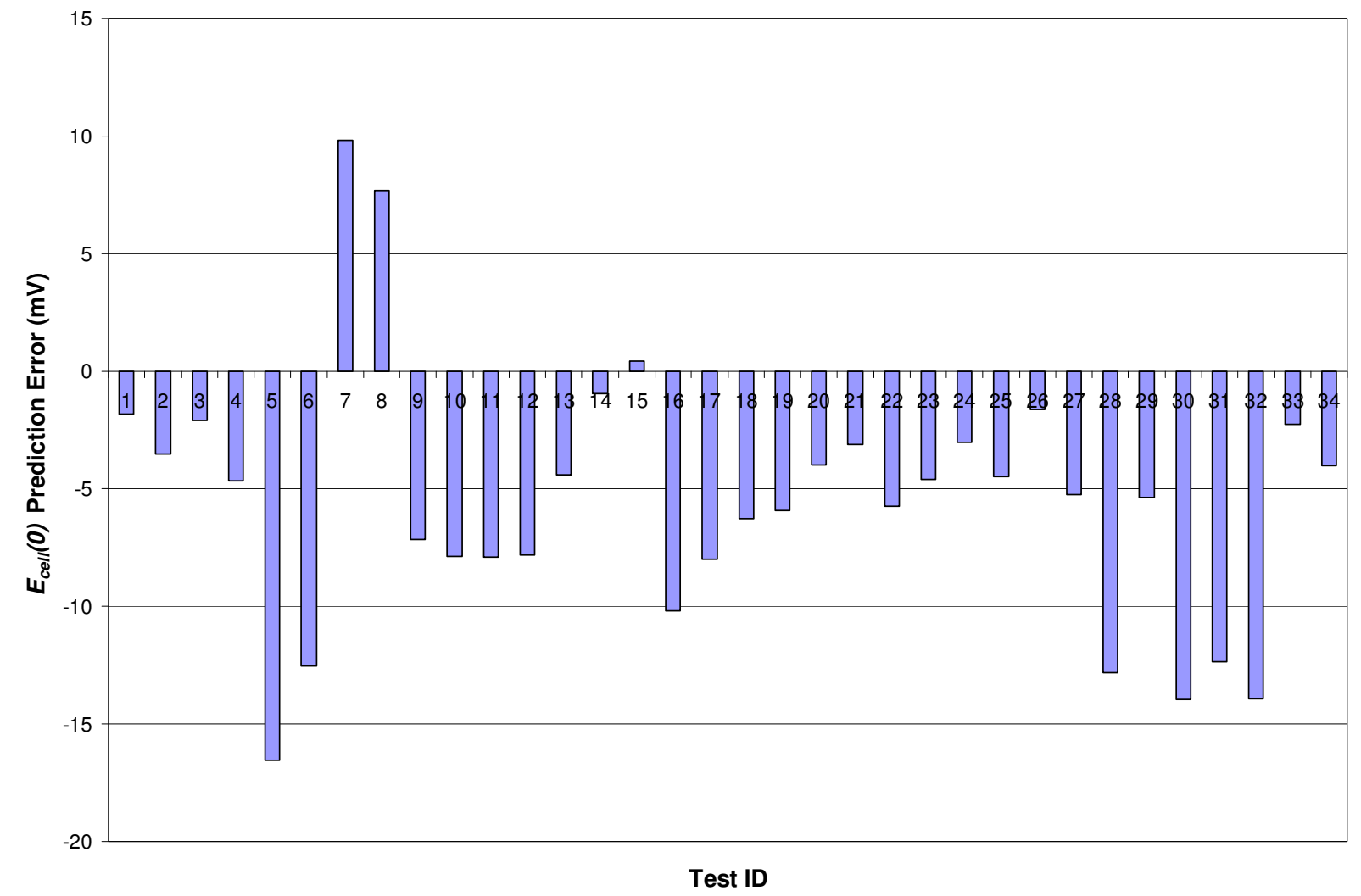

Figure 19 Calculated $E_{\text {cell }}(0)$ error magnitude for all 34 test cases.

A summary of overall numerical error is provided in Table 3 below and confirms that it was possible to validate a set of empirical model constants for LGFCS SOFC technology

Table 3 Summary of numerical errors for penta cell tests.

\begin{tabular}{|l|l|l|}
\hline Numerical Prediction & Mean Error $(\%)$ & Maximum Error $(\%)$ \\
\hline$E_{\text {cell }}(0)$ & 0.7 & 1.8 \\
\hline$E_{\text {cell }}\left(i_{\max }\right)$ & 1.8 & 7.0 \\
\hline$A S R$ & 6.0 & 20.0 \\
\hline
\end{tabular}

\subsection{SUBSTRATE MODEL VALIDATION}

The components required for a substrate model are shown in Figure 20. 


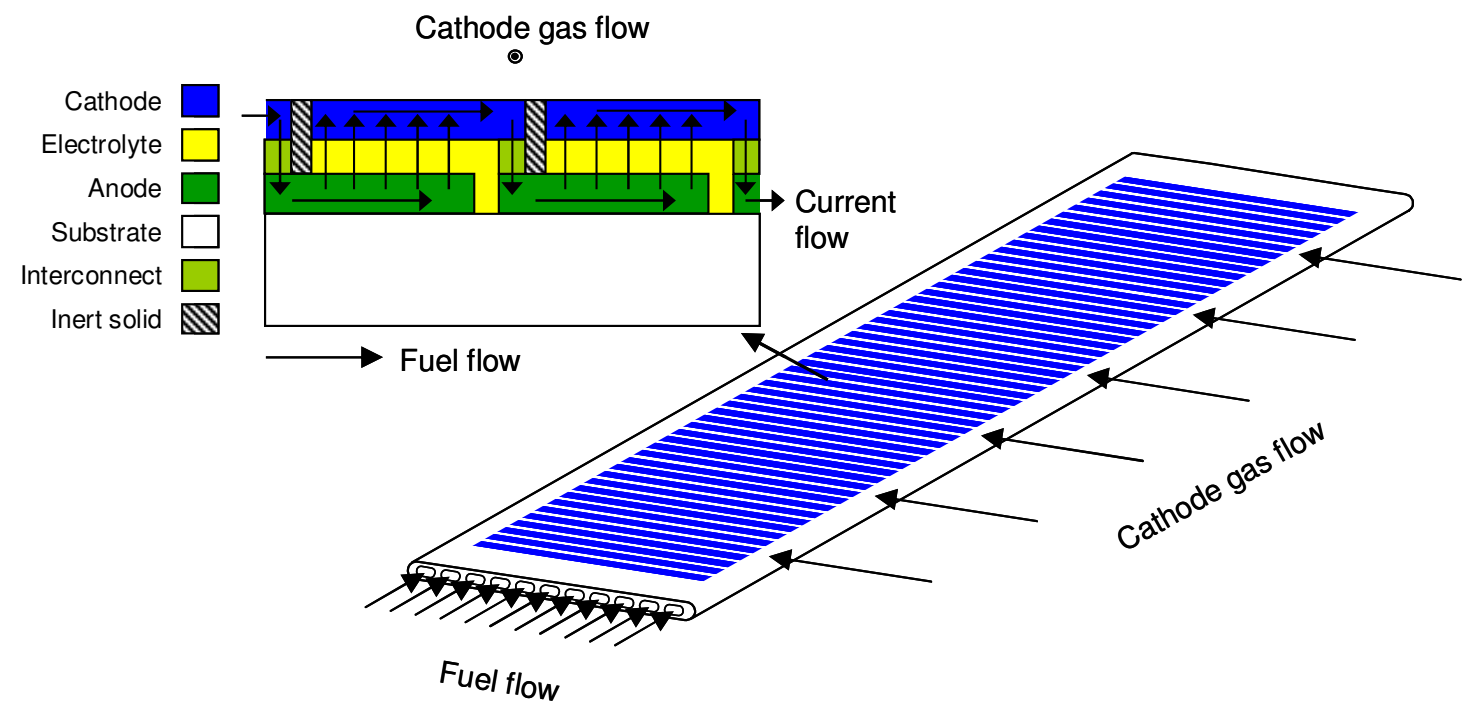

Figure 20 Thee-dimensional model of substrate.

The model configuration and computational mesh used in STAR-CCM+ to represent these components is shown in Figure 21.

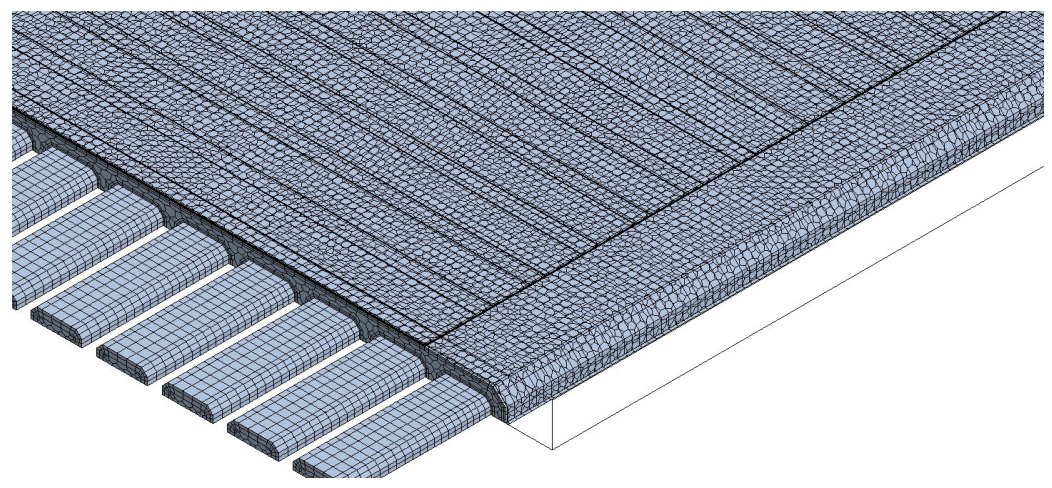

Figure 21 Close up of a STAR-CCM+ substrate model mesh.

Figure 21 shows the level of detail used to model each cell on the substrate and overall this model contains around 1.5 million grid cells and represents the upper part of the substrate only.

The following physical models were installed using the GUI to create a realistic substrate model:

- High fidelity fluid flow to describe the flow of gases in the porous cell components and the adjacent gas channels.

- High fidelity porous flow model to provide a complete description of mass transport in the porous cell components and enhance the diffusion calculation in the adjacent gas channels. Material properties for this model were obtained from specific tests on individual cell components.

- Low fidelity thermal model to ensure isothermal operation. 
- Low fidelity chemistry model to capture the behavior of the water gas shift reaction $\mathrm{H}_{2} \mathrm{O}+\mathrm{CO} \longleftrightarrow \mathrm{CO}_{2}+\mathrm{H}_{2}$ which is catalyzed by the anode material. Kinetic constants for this reaction were obtained from previous studies [2].

- High fidelity current flow model to numerically simulate the current flow through each cell component.

- High fidelity electrochemistry to integrate with the high fidelity current flow model and to specify the following electrochemical reactions at the cathode and anode/electrolyte interfaces respectively:

$$
\begin{array}{ll}
\circ & \mathrm{O}_{2}+2 \mathrm{~V}_{\mathrm{O}}^{\bullet \bullet}(\mathrm{YSZ})+4 \mathrm{e}^{-} \longleftrightarrow 2 \mathrm{O}_{\mathrm{O}}^{\mathrm{X}}(\mathrm{YSZ}) \\
\circ & \mathrm{H}_{2}+\mathrm{O}_{\mathrm{O}}^{\mathrm{X}}(\mathrm{YSZ}) \longleftrightarrow \mathrm{H}_{2} \mathrm{O}+2 \mathrm{e}^{-}+\mathrm{V}_{\mathrm{O}}^{\bullet \bullet}(\mathrm{YSZ})
\end{array}
$$

The kinetic constants required for the electrochemistry models were determined from other validation work performed by LGFCS.

- Low fidelity time model for steady-state calculations.

This model was run using a wide range of prescribed operating conditions including: pressure, temperature, fuel composition $\left(\mathrm{H}_{2}, \mathrm{H}_{2} \mathrm{O}, \mathrm{CO}, \mathrm{CO}_{2}, \mathrm{~N}_{2}\right)$, air composition $\left(\mathrm{N}_{2}, \mathrm{O}_{2}\right)$ and current density to validate the predicted cell potentials against the available experimental data.

Figure 22 shows a typical plot of hydrogen mole fraction within the fuel side cell components and illustrates the change in fuel composition caused by the electrochemical consumption of hydrogen along the fuel flow path.

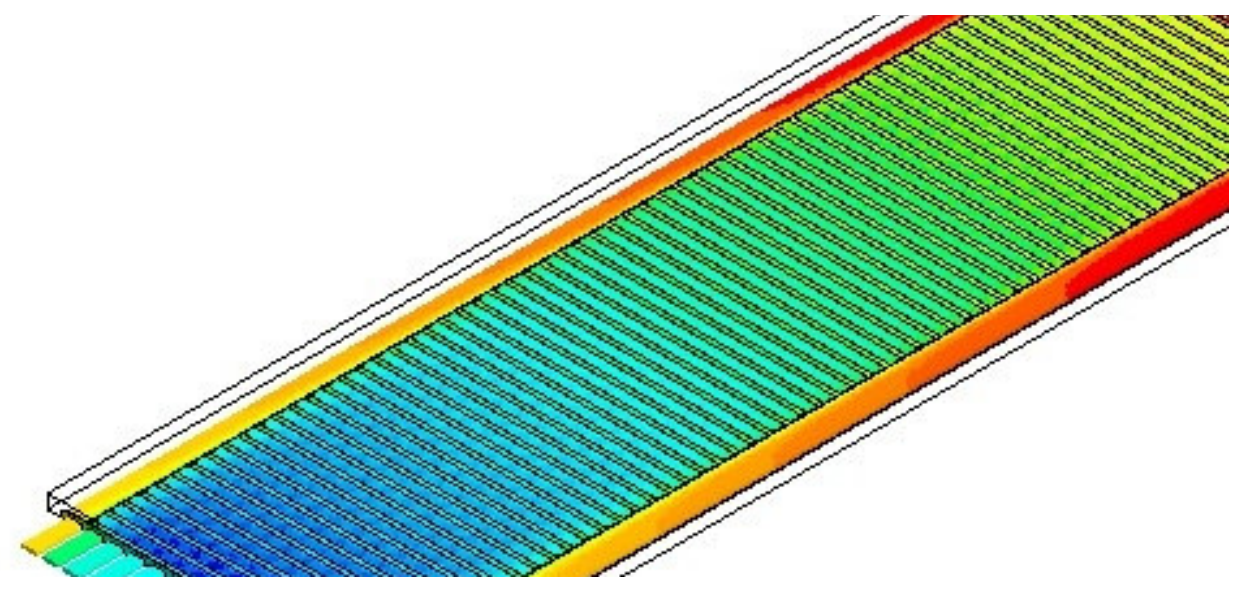

Figure 22 Hydrogen mole fraction distribution in the fuel side cell components.

The substrate model also provides detailed information about the electrochemical reactions that proceed in each cell fuel cell and Figure $\mathbf{2 3}$ shows the variation in current density in each electrolyte. 


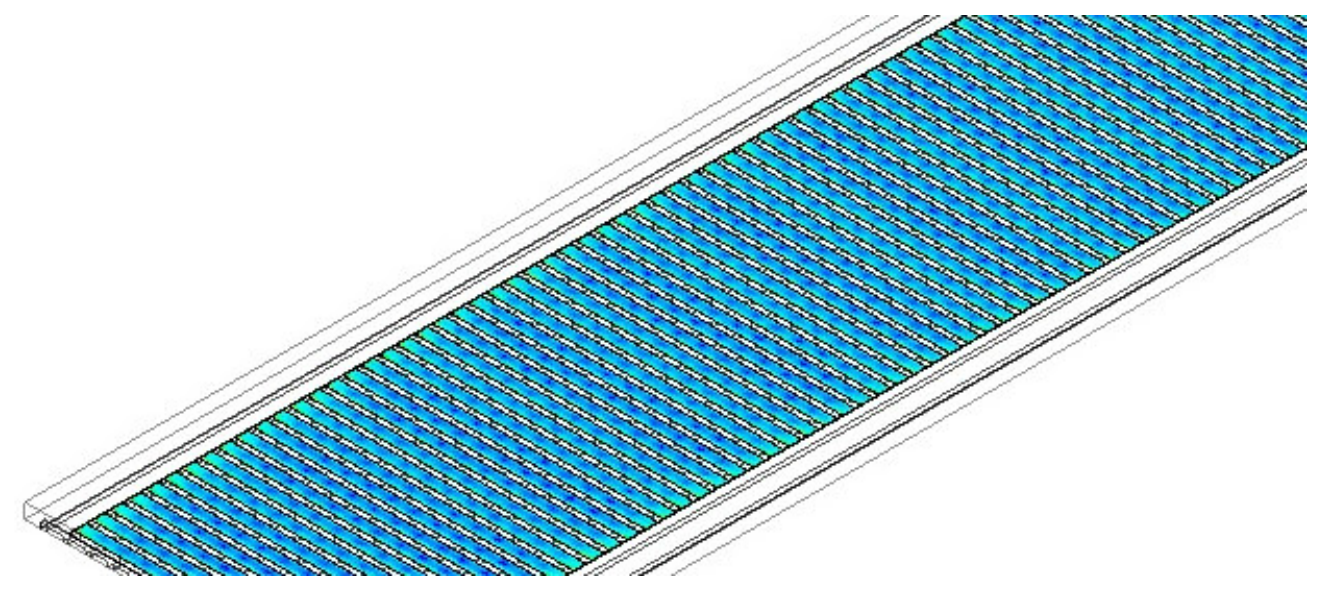

Figure 23 Calculated current density distribution in each electrolyte of a substrate.

Small variations in current density are observed as a result of the transport characteristics of the substrate geometry and the current flow path through the cell components.

Several comparisons were performed against experimental data and comparisons between measured current-voltage (IV) behavior. Numerical predictions were within an acceptable level of accuracy, typically $\pm 6 \%$ of the measured value.

\subsection{BUNDLE MODEL VALIDATION}

The components required for a bundle model are shown in Figure 24.

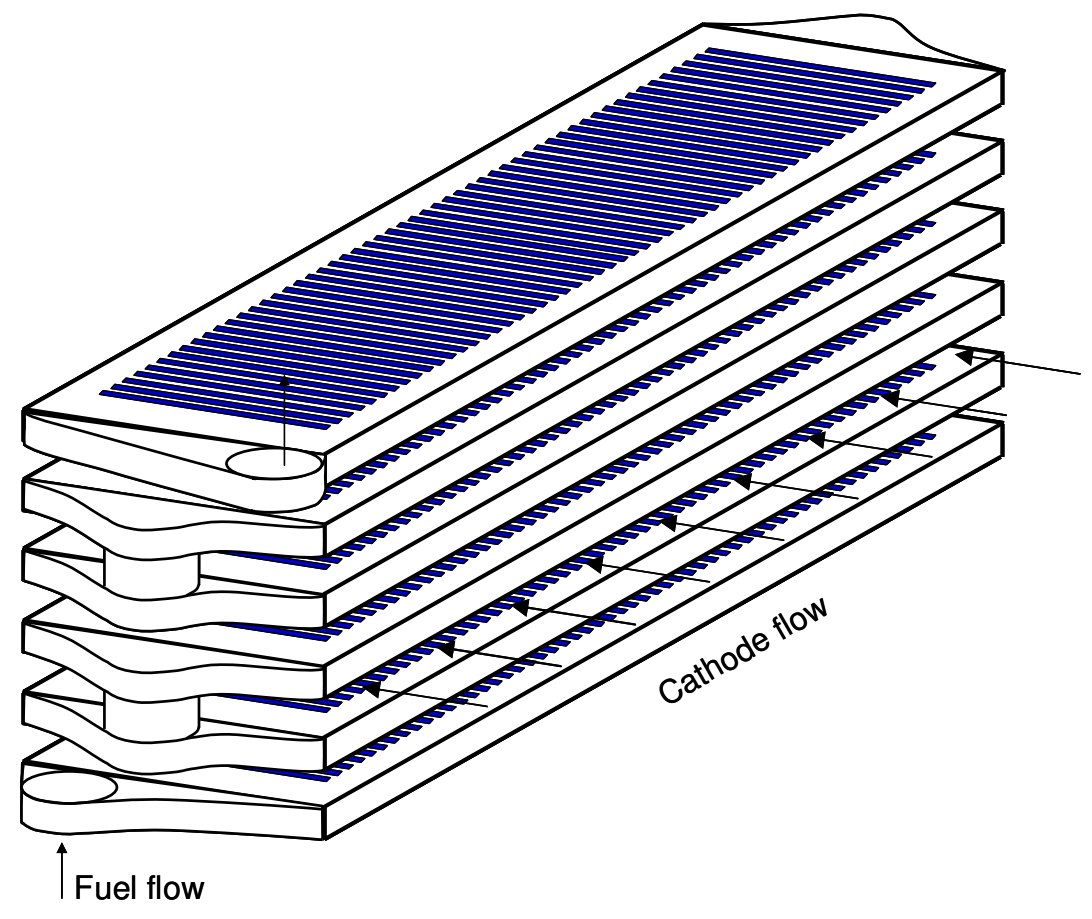

Figure 24 Thee-dimensional model of bundle. 
The mesh required for this model contains around 8 million grid cells and represents all the bundle components shown in Figure 24.

The following physical models were installed using the GUI to create a realistic bundle model:

- High fidelity fluid flow to describe the flow of gases in the porous cell components and the adjacent gas channels.

- High fidelity porous flow model to provide a complete description of mass transport in the porous cell components and enhance the diffusion calculation in the adjacent gas channels. Material properties for this model were obtained from specific tests on individual cell components.

- Low fidelity thermal model to ensure isothermal operation.

- Low fidelity chemistry model to capture the behavior of the water gas shift reaction $\mathrm{H}_{2} \mathrm{O}+\mathrm{CO} \longleftrightarrow \mathrm{CO}_{2}+\mathrm{H}_{2}$ which is catalyzed by the anode material. Kinetic constants for this reaction were obtained from previous studies [2].

- Low fidelity current flow model to use an analytical model of the current flowing through the cell components.

- Low fidelity electrochemistry model to integrate with the low fidelity current flow model and to specify the following electrochemical reactions at the cathode and anode/electrolyte interfaces respectively:

$$
\begin{array}{ll}
\circ & \mathrm{O}_{2}+2 \mathrm{~V}_{\mathrm{O}}^{\bullet \bullet}(\mathrm{YSZ})+4 \mathrm{e}^{-} \longleftrightarrow 2 \mathrm{O}_{\mathrm{O}}^{\mathrm{X}}(\mathrm{YSZ}) \\
\circ & \mathrm{H}_{2}+\mathrm{O}_{\mathrm{O}}^{\mathrm{X}}(\mathrm{YSZ}) \longleftrightarrow \mathrm{H}_{2} \mathrm{O}+2 \mathrm{e}^{-}+\mathrm{V}_{\mathrm{O}}^{\bullet \bullet}(\mathrm{YSZ})
\end{array}
$$

The kinetic constants required for the electrochemistry models were determined from other validation work performed by LGFCS.

- Low fidelity time model for steady-state calculations.

This model was run using a wide range of prescribed operating conditions including: pressure, temperature, fuel composition $\left(\mathrm{H}_{2}, \mathrm{H}_{2} \mathrm{O}, \mathrm{CO}, \mathrm{CO}_{2}, \mathrm{~N}_{2}\right)$, air composition $\left(\mathrm{N}_{2}, \mathrm{O}_{2}\right)$ and current density to validate the predicted cell potentials against the available experimental data.

Figure 25 shows a typical plot of hydrogen mole fraction within the fuel side bundle components and illustrates the change in fuel composition caused by the electrochemical consumption of hydrogen along the fuel flow path.

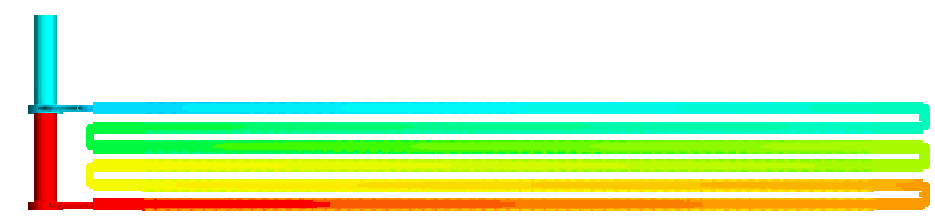

Figure 25 Hydrogen mole fraction distribution in the fuel side bundle components

Several IV curve comparisons have been made against experimental bundle measurements and the numerical errors are within acceptable levels, typically $\pm 8 \%$ of the measured value. 


\subsection{LOW FIDELITY EMPIRICAL DEGRADATION MODEL VALIDATION}

The empirical degradation model was built and validated against six sets of cell degradation data measured on five-cell test pieces with over 4,000 hours of operation. Several time dependent variables were identified from the measurements:

- Electrolyte conductivity.

- Interconnect resistance.

- Anode activation pre-exponential term.

- Cathode activation pre-exponential term.

Model constants were calculated empirically to produce a low fidelity degradation model for each time dependent variable. Degradation models were installed for each variable into an existing penta cell model, see Section 5.2, using the GUI. Comparisons were made between measured and predicted current-voltage (IV) behavior for 48 different test cases representing different experimental test conditions and times on test.

Numerical errors were calculated by comparing experimental data and numerical predictions for three different performance measurements: $E_{\text {cell }}(0), E_{\text {cell }}\left(i_{\max }\right)$ and $A S R$, see Section 5.1. Figure 26 shows a plot of calculated $E_{\text {cell }}(0)$ numerical errors for the 48 test cases considered and illustrates the typical level of numerical error obtained from MPC calculations including empirical degradation models.

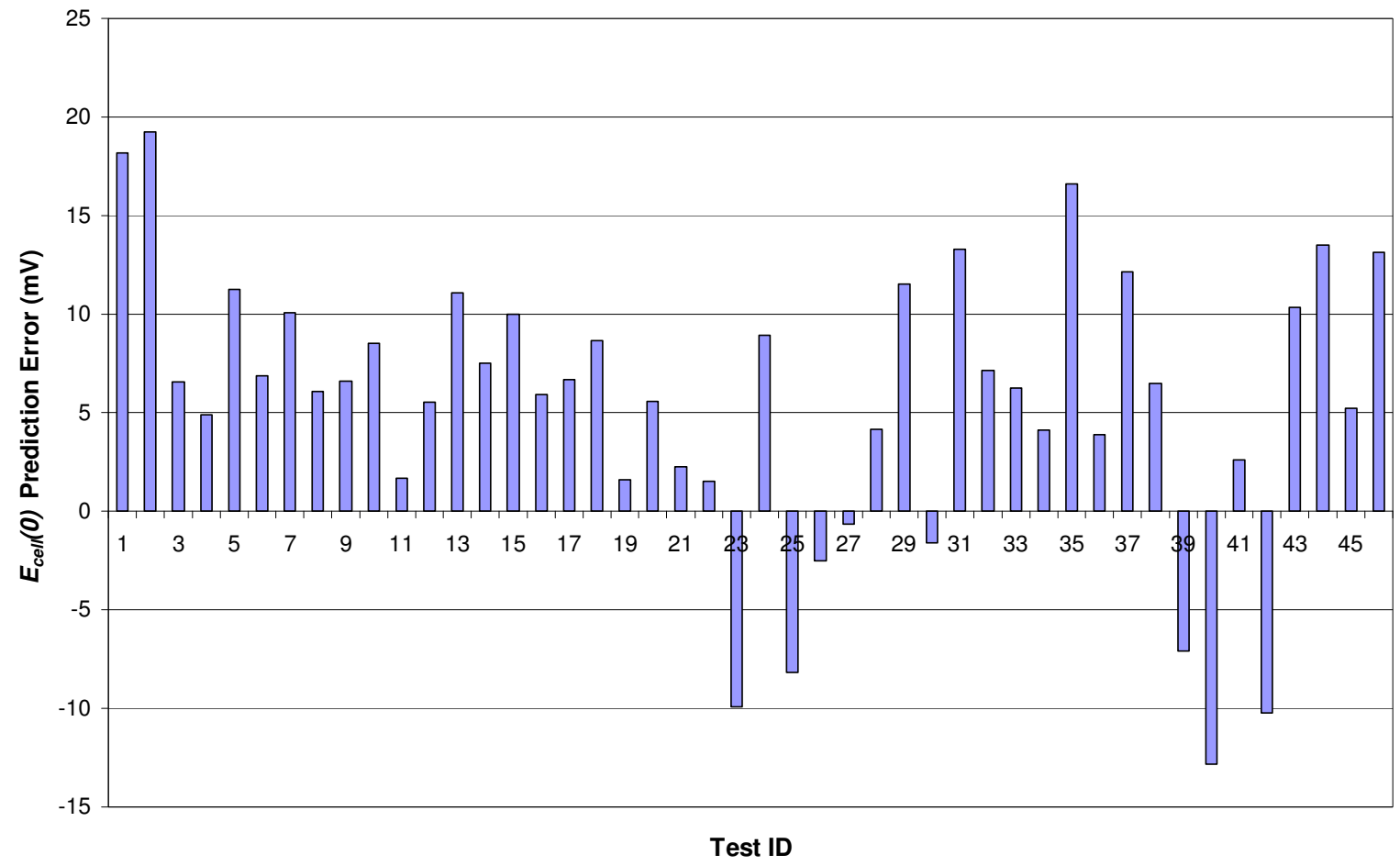

Figure 26 Calculated $E_{c e l l}(0)$ error magnitude for all 48 test cases. 
A summary of overall numerical error is provided in Table $\mathbf{4}$ below and an acceptable level of accuracy for a comparison against several tests obtained over a 4,000 hour period

Table 4 Summary of numerical errors for penta cell tests obtained over a 4,000 hour period.

\begin{tabular}{|l|l|l|}
\hline Numerical Prediction & Mean Error $(\%)$ & Maximum Error $(\%)$ \\
\hline$E_{\text {cell }}(0)$ & 0.9 & 2.0 \\
\hline$E_{\text {cell }}\left(i_{\max }\right)$ & 3.2 & 15.1 \\
\hline$A S R$ & 10.5 & 37.6 \\
\hline
\end{tabular}

\subsection{HIGH FIDELITY KINETIC DEGRADATION MODEL DEMONSTRATION}

A kinetic degradation model has been built to qualitatively assess the MPC ability to capture observed degradation phenomena. A well documented degradation case, "SOFC LSM:YSZ cathode degradation induced by moisture: An impedance spectroscopy study" [5] was chosen from the literature to assess this model. In this study the authors suggest several processes that may contribute to the observed reduction in cathode performance:

- Diffusion of a Manganese species from LSM particles along YSZ boundaries leading to an initial improvement in cathode performance.

- Reaction of Manganese with steam to form an unknown gaseous species and remove Manganese from the system.

- Gradual depletion of the amount of Manganese remaining in the LSM particles until contact with surrounding YSZ is reduced and performance is permanently lost.

These processes were modeled with two time dependent variables and one reaction mechanism to calculate the rate at which manganese reacts with steam. Assumptions were made about the unknown quantities in the models.

The model was used to calculate cell potential over a period of time during which a cell was run with air with a controlled level of humidity ranging from dry to highly humidified ( $6 \%$ water vapor content). A comparison with the published experimental data showed that the kinetic degradation model was able to qualitatively capture several significant aspects of this degradation process including:

- An initial improvement in performance as Manganese spreads from the LSM particles to cover the YSZ surface.

- A sudden degradation in performance when steam is introduced to the cathode.

- A steady and permanent decline in performance in the presence of steam over a long period of time.

- A recovery of some performance when steam is removed and the cathode is fed dry air.

This test case successfully demonstrated the ability of the kinetic degradation MPC feature to qualitatively model published degradation behavior for a system involving several physical processes.

\subsection{DEMONSTRATION OF MPC TRANSIENT CAPABILITIES (LARGE TIME STEPS)}

Transient calculations were tested on a range of models from single cell to bundle scale; results are presented below for a transient substrate model. The components required for this model are shown in Figure 27. 


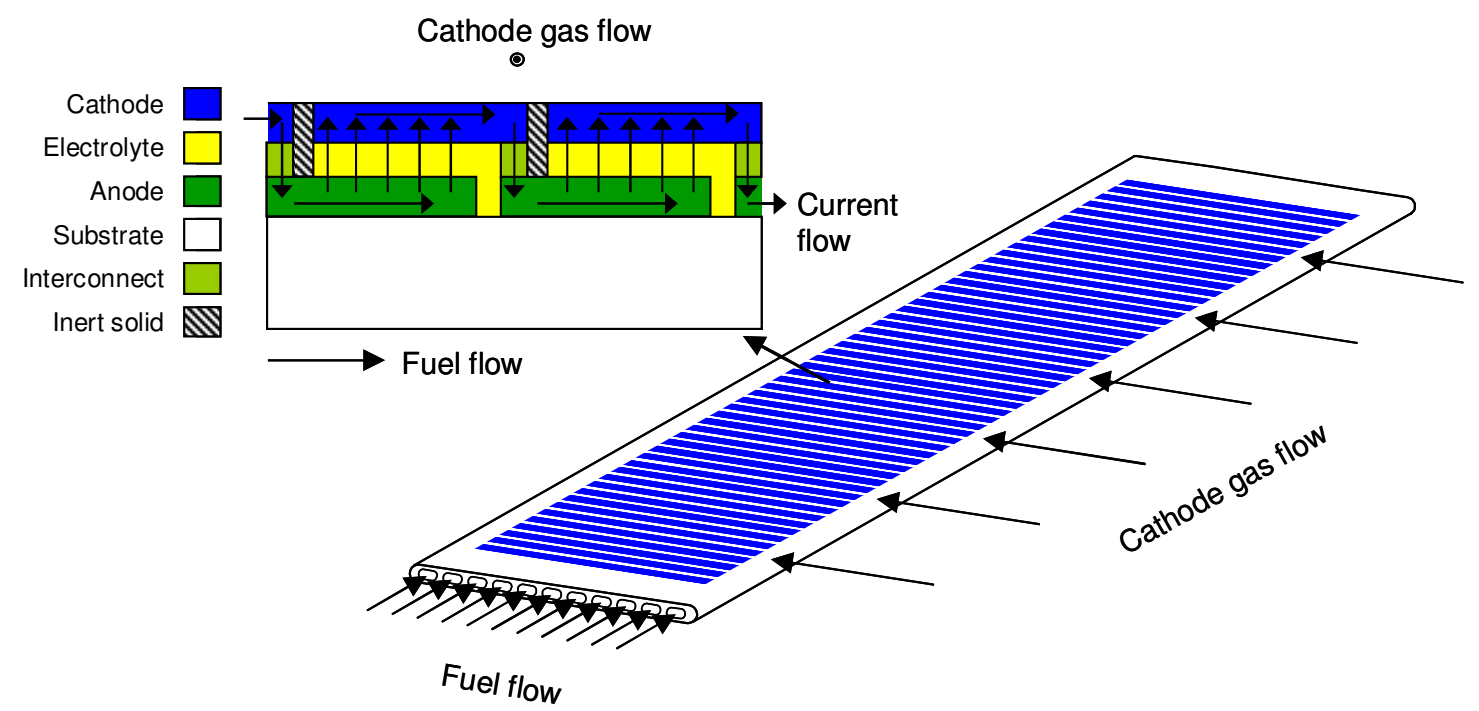

Figure 27 Thee-dimensional model of substrate.

The model configuration and computational mesh used in STAR-CCM+ to represent these components is shown in Figure 28.

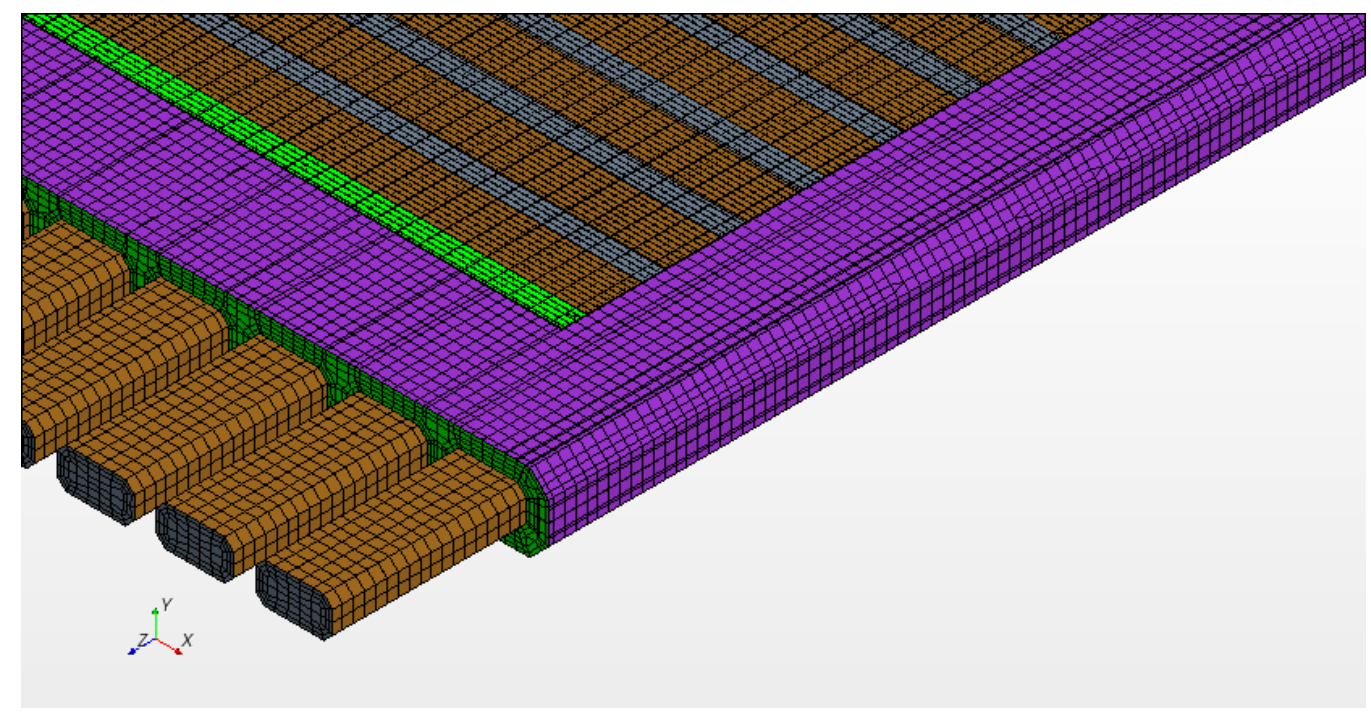

Figure 28 Close up of a STAR-CCM+ substrate model

Figure 28 shows the level of detail used to model each cell on the substrate with the overall model containing around 4.4 million grid cells.

The following physical models were installed using the GUI to create a realistic substrate model:

- High fidelity fluid flow to describe the flow of gases in the porous cell components and the adjacent gas channels. 
- High fidelity porous flow model to provide a complete description of mass transport in the porous cell components and enhance the diffusion calculation in the adjacent gas channels. Material properties for this model were obtained from specific tests on individual cell components.

- Low fidelity thermal model to ensure isothermal operation.

- Low fidelity chemistry model to capture the behavior of the water gas shift reaction $\mathrm{H}_{2} \mathrm{O}+\mathrm{CO} \longleftrightarrow \mathrm{CO}_{2}+\mathrm{H}_{2}$ which is catalyzed by the anode material. Kinetic constants for this reaction were obtained from previous studies [2].

- High fidelity current flow model to numerically simulate the current flow through each cell component.

- High fidelity electrochemistry to integrate with the high fidelity current flow model and to specify the following electrochemical reactions at the cathode and anode/electrolyte interfaces respectively:

$$
\begin{array}{ll}
\circ & \mathrm{O}_{2}+2 \mathrm{~V}_{\mathrm{O}}^{\bullet \bullet}(\mathrm{YSZ})+4 \mathrm{e}^{-} \longleftrightarrow 2 \mathrm{O}_{\mathrm{O}}^{\mathrm{X}}(\mathrm{YSZ}) \\
\circ & \mathrm{H}_{2}+\mathrm{O}_{\mathrm{O}}^{\mathrm{X}}(\mathrm{YSZ}) \longleftrightarrow \mathrm{H}_{2} \mathrm{O}+2 \mathrm{e}^{-}+\mathrm{V}_{\mathrm{O}}^{\bullet \bullet}(\mathrm{YSZ})
\end{array}
$$

The kinetic constants required for the electrochemistry models were determined from other validation work performed by LGFCS.

- High fidelity time model to allow time dependent calculations.

This model was run over a series of time steps to simulate a sudden change in fuel composition from a nominal fuel mixture $\left(\mathrm{H}_{2}, \mathrm{H}_{2} \mathrm{O}, \mathrm{CO}, \mathrm{CO}_{2}, \mathrm{~N}_{2}\right)$ to one with a depleted amount of hydrogen. Figure 29 illustrates the steady-state distribution of hydrogen mole fraction in the substrate under nominal operating conditions.

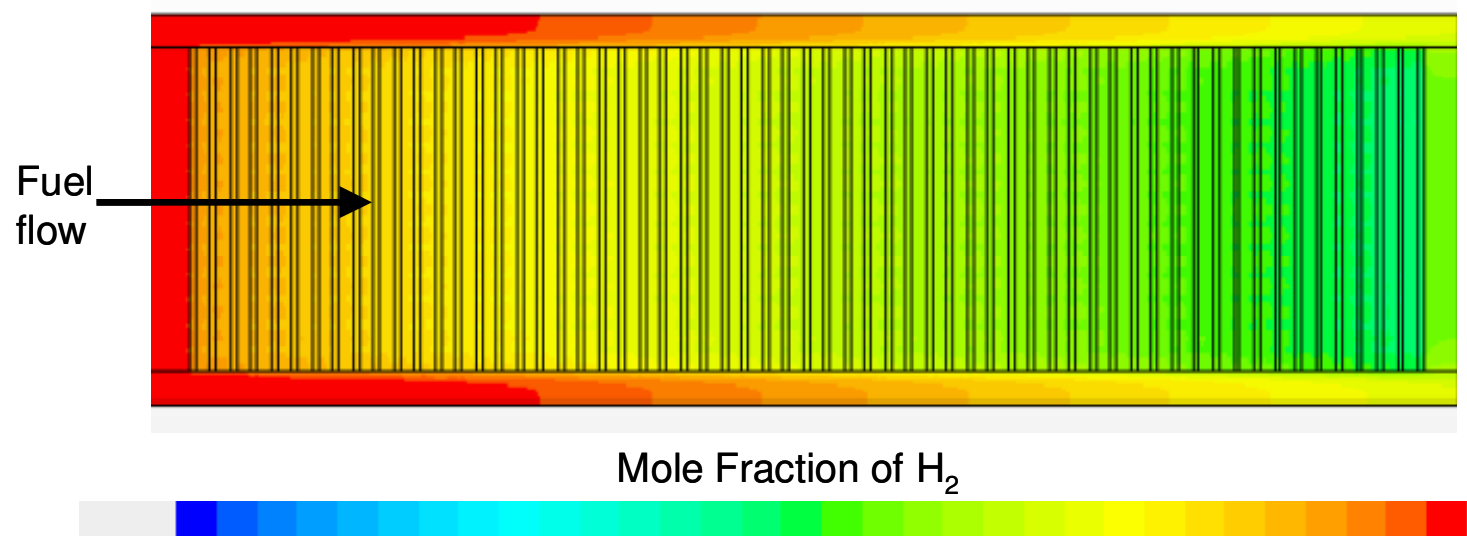

Figure 29 Steady state distribution of hydrogen mole fraction within a three-dimensional substrate model.

After reaching the steady-state, the inlet fuel hydrogen composition was reduced by $50 \%$ in a step change. Figure 30 and Figure 31 show the transient hydrogen distribution calculated after 0.1 and 0.2 seconds following the step change. 


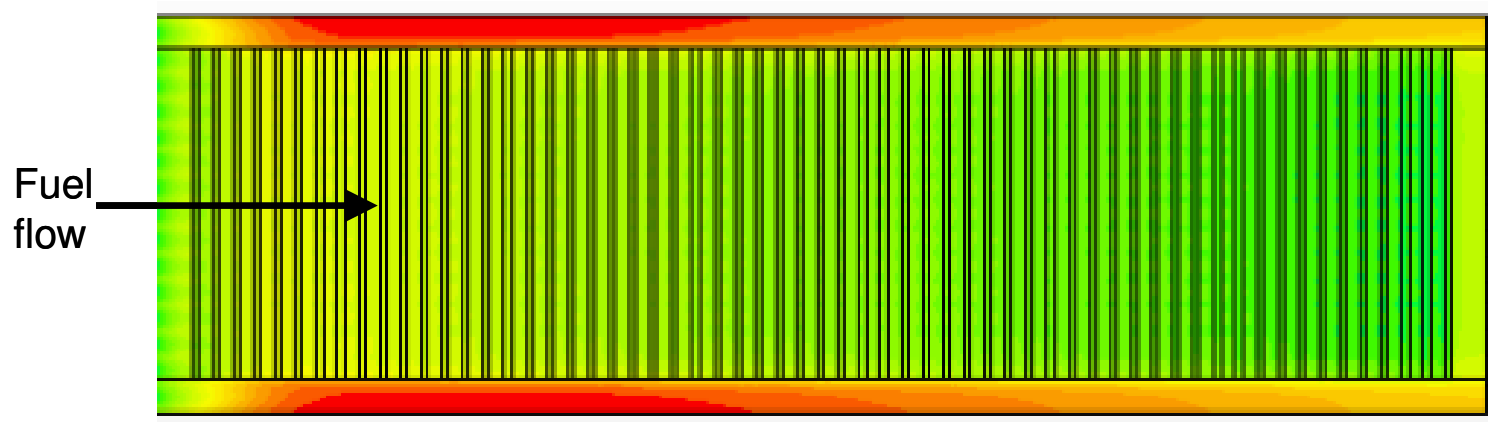

Figure 30 Transient distribution of hydrogen mole fraction 0.1 seconds after halving inlet fuel hydrogen composition.

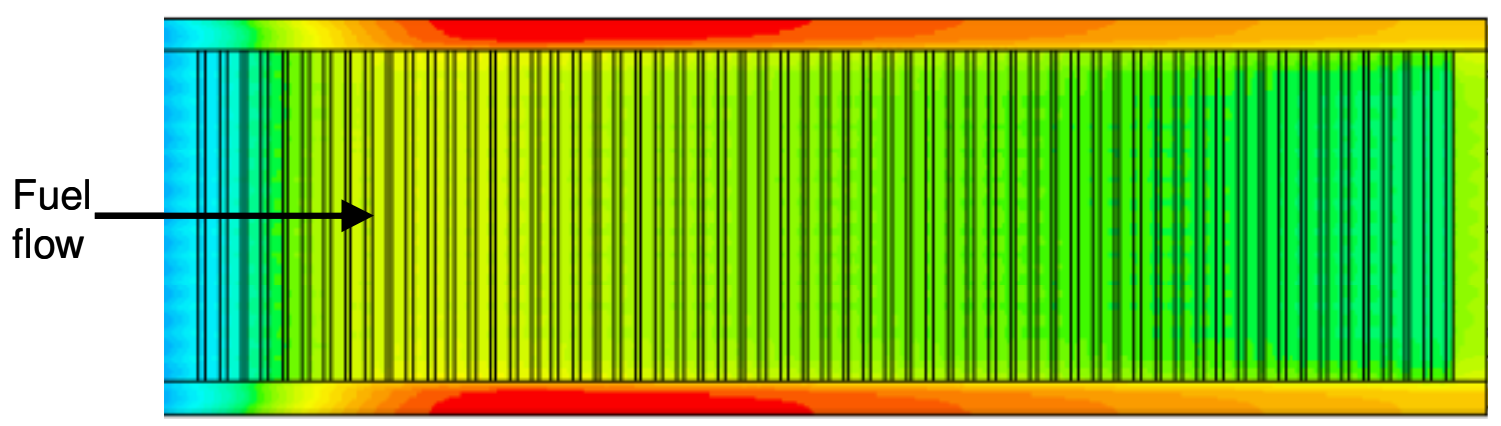

Figure 31 Transient distribution of hydrogen mole fraction 0.2 seconds after halving inlet fuel hydrogen composition.

The tests performed were able to successfully demonstrate the MPC transient capabilities for large time steps.

\subsection{DEMONSTRATION OF MPC TRANSIENT CAPABILITIES (SMALL TIME STEPS)}

The MPC transient capability for very small time steps was tested by analyzing the effect on cell performance of ripples in the applied current. A two-dimensional model of a five cell test piece was built for this task, see Figure 32.

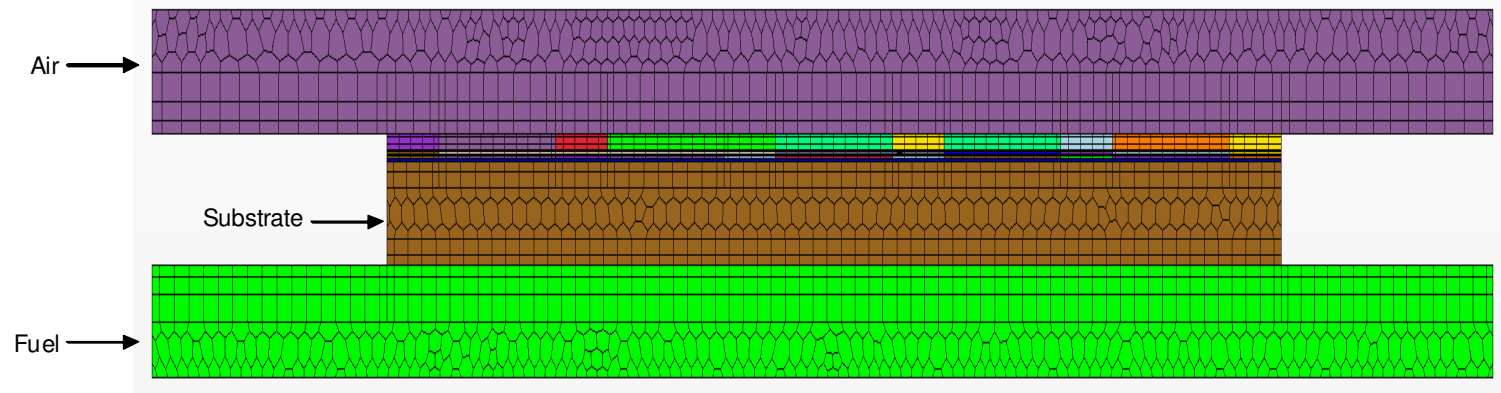

Figure 32 Two-dimensional model of five cell test piece. 
This model includes five fuel cells fabricated on the outer surface of the substrate, an internal fuel channel, an external air flow channel and all the cell components necessary to model current flow and electrochemistry. Similar physical models to those described in Section 5.2 were installed and a series of transient simulations were performed. A time step size of $1 / 480 \mathrm{~s}$ was used to model an applied current with a $60 \mathrm{~Hz}$ sinusoidal ripple (10\% peak to trough magnitude). Figure 33 shows the calculated average cell voltage response of the cells to the input current signal.

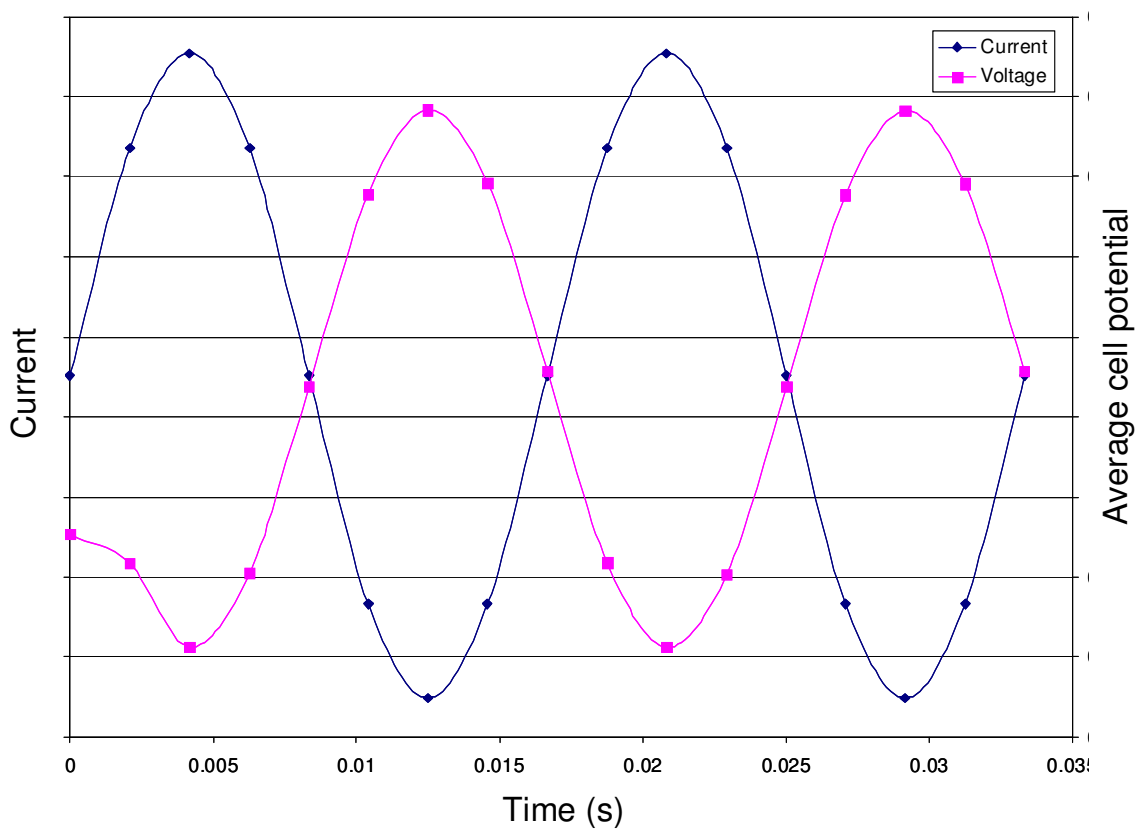

Figure 33 Voltage response of cells to sinusoidal input current signal

Figure 33 shows that there is a phase shift between input and output signals which is caused by the slower response of the diffusion processes in the porous components to the input signal. Further details of this are given in Figure $\mathbf{3 4}$ which shows the response of minimum hydrogen mole fraction at the anode/electrolyte interface to the input current signal. 


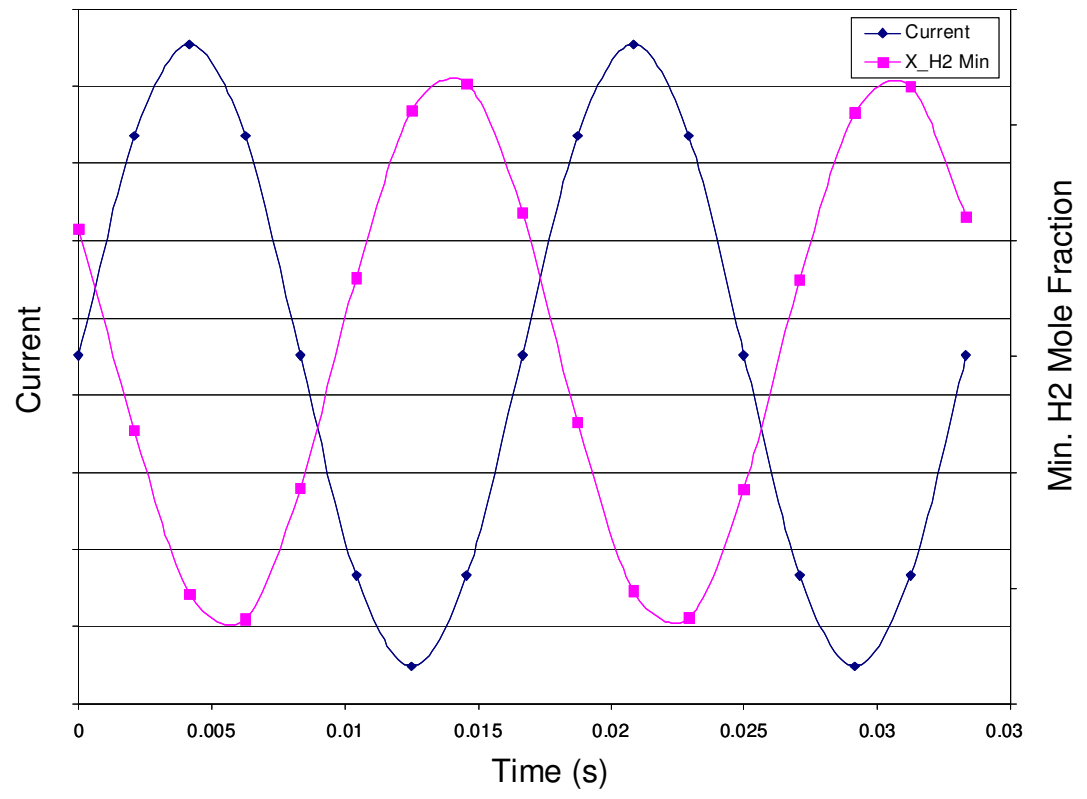

Figure 34 Anode/Electrolyte hydrogen mole fraction response to sinusoidal input current signal.

Further validation of the predicted signal responses against cell impedance measurements is outside the scope of this project.

\section{ADVANCED ENGINEERING APPLICATIONS}

During this project the predictive capabilities of the MPC were tested in a variety of advanced engineering applications. Feedback from these tests was used to improve the MPC modeling capabilities and simulation methodology.

\subsection{STRIP FUEL DISTRIBUTION}

The purpose of this application was to verify the design point fuel distribution in a strip by building a detailed geometric model of all the fuel flow components. Simplifications were made to the representation of each bundle and several cases were analyzed. Figure 35 shows a typical plot of the hydrogen mole fraction distribution obtained from one of these strip calculations. 


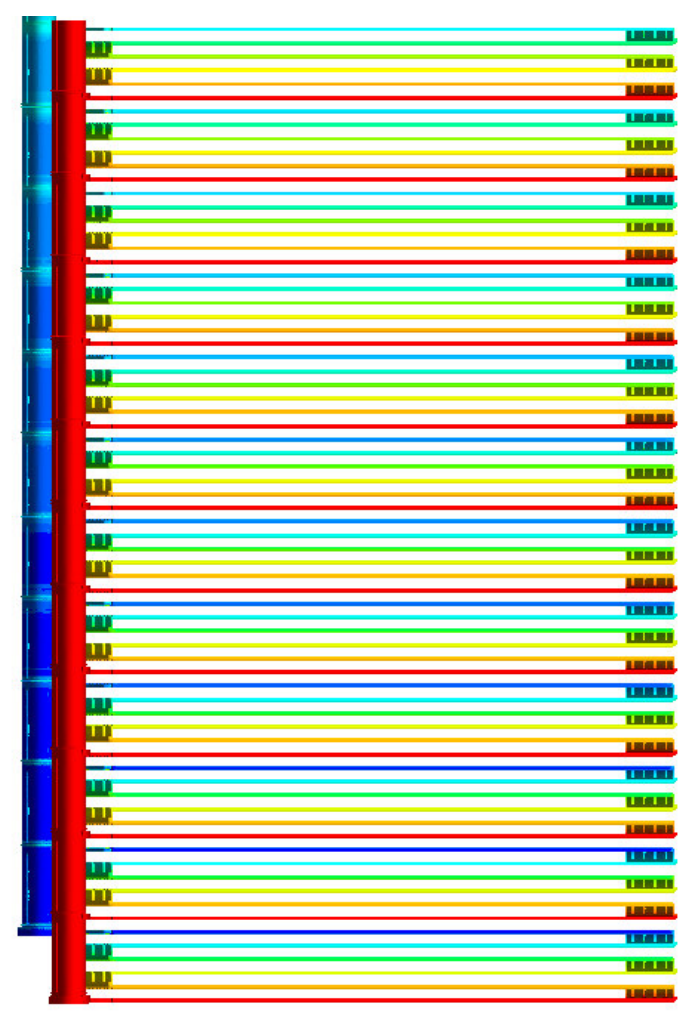

Mole Fraction of $\mathrm{H}_{2}$

Figure 35 Predicted fuel composition in a strip.

The MPC predictions from this model were validated against experimental measurements taken from a specially commissioned test rig and further calculation results were used to verify the design point fuel distribution.

\subsection{DETAILED ANALYSIS OF SUBSTRATE ELECTRICAL CONNECTIONS}

This application focused on the design of the interconnect at the start and end of each row of cells to understand the effect their design has on substrate and cell performance. A model of a substrate was built including the geometric details of these interconnect regions and similar physical models to those described in Section 5.7 were installed using the GUI.

Several test cases were run to analyze the effect of failures and design changes on overall performance. The model predictions were used to inform analysis of experimental data and discussions on improvements to substrate design.

\subsection{THERMAL TRANSIENT ANALYSIS}

This application was focused on analyzing the interaction between fluid flow and temperature distribution during a block cool down process. A simplified model of two bundles was built, see Figure 36. 


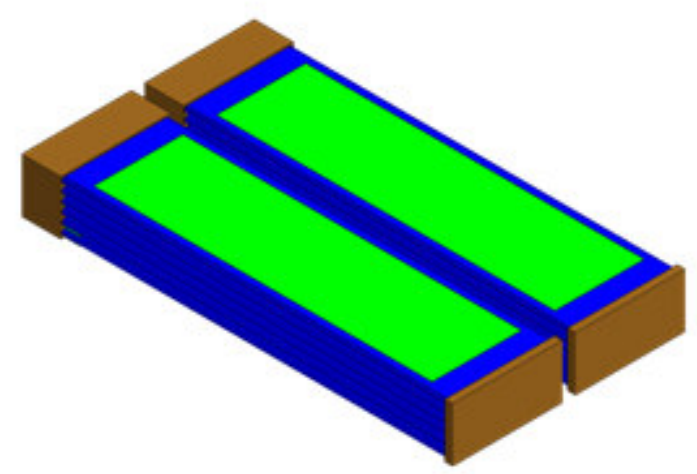

Figure 36 A simplified model of two bundles.

Several calculations were performed over time step sizes ranging from less than 1 second to several minutes. Results from these predictions were compared with other models and were used to support design analysis tasks.

\subsection{THERMAL ANALYSIS OF BUNDLE OPERATION WITHIN A BLOCK}

This application focused on making detailed predictions of temperature distributions within the stack components for input to stress analysis calculations. It is not practical to build a detailed model of a whole block and instead temperature distributions from a less detailed block simulation were used as boundary conditions for the detailed MPC bundle model. This model considered all the components in a bundle including substrates and fuel pipes and manifolds; it contained a total of 9 million grid cells. This model was used to perform detailed thermal analysis at different bundle locations within the block and generate temperature distributions for stress analysis.

\subsection{DETAILED ANALYSIS OF BUNDLE ELECTRICAL CONNECTIONS}

This application focused on the design and configuration of electrical connections between substrates in a bundle. A model of two substrates was built using settings and geometry similar to that used in previous analysis tasks, see Section 6.2. Electrical connections were included between the rows of cells on each of the substrates. A range of different configurations and possible incorrect connections were analyzed and the calculated results were used to inform design activities.

\subsection{DETAILED GEOMETRIC MODELING OF CELL COMPONENTS}

This application focused on developing a realistic model of cell component layout including all printed components with the goal to develop a more detailed understanding of cell design considerations. Until this point it was common practice to simplify a cell model to just three layers (anode/electrolyte/cathode) to reduce the complexity of MPC models. Several improvements were made to the MPC methodology during the final year of this project with the specific aim of managing the high level of geometric complexity introduced into this model.

\section{TESTING OF MPC WITH ENGINEERING STAFF}

The MPC is intended for use by engineering staff outside of the project team and the MPC project strategy has included the development of a GUI to meet this requirement, see Section 3. The design of 
the GUI was completed during the final year of this project with several features intended to aid the nonexpert user:

- There are several tabbed pages in a structured layout arranged to provide a step by step approach to model building.

- A message window provides a summary of any errors and useful tips when a task is performed.

- A set of system status checks is performed prior to executing each task to determine if any issues exist within the model. Any errors or problems are reported (along with useful tips) in the GUI message window.

- A list of acceptable entries is stored for every EMS cell entry that accepts a user input. All EMS inputs are tested prior to being used and any errors reported back to the user with an explanation of the acceptable inputs.

A detailed user manual and a set of relevant tutorials were also prepared in addition to the GUI features described above. Several training classes have been given to new users and there are currently eight engineering staff using the MPC both full time and part time.

The MPC is now being used to support a range of activities within the business that include SOFC and non-SOFC design analysis applications.

\section{CONCLUSION}

This report has presented a summary of the work performed during the LG Solid Oxide Fuel Cell (SOFC) Model Development project (contract DE-FE0000773) which started on October 1, 2009 and was completed on March 31, 2013. The aim of this project was for LGFCS to develop a multi-physics solid oxide fuel cell (SOFC) computer code (MPC) for performance calculations of its fuel cell structure to support fuel cell product design and development.

During the early phases of this project an extensive review of published SOFC modeling research was initiated in addition to an in-house review to determine the key MPC functional requirements. These requirements included physical modeling capabilities and functional capabilities such as being suitable for use by engineering staff outside of the project team. A downselect exercise was then undertaken and STAR-CCM+ (CD-adapco) was identified as the most suitable commercially available code for use as the MPC. A project strategy was developed to leverage the custom modeling capabilities of STAR-CCM+ to build a simulation tool capable of performing advanced SOFC simulations and be used by engineering staff outside of the project team. This strategy involved storing all model data in a Microsoft Excel spreadsheet and installing a custom Graphical User Interface (GUI) into STAR-CCM+ to read the model data and automatically perform all of the tasks required to pre-process, execute and post-process simulations.

The remainder of this project was split into two concurrent work streams: i) the installation of existing modeling capabilities and code enhancements into the GUI to meet the physical model requirements for MPC SOFC simulations and ii) the verification and validation of LGFCS SOFC cell technology models using the latest experimental data. The program of work for these work streams was planned around the delivery of eight code releases at intervals throughout the project. A summary is reported herein of the available MPC physical modeling capabilities and the completed model verification and validation tasks.

The GUI provides a set of interchangeable physical models (fluid flow, porous flow, heat transfer, current flow, chemistry, electrochemistry, degradation and time) that can be installed to customize the MPC to 
meet specific application dependent requirements. Two fidelity levels are provided for each of the available models and these are discussed in detail in terms of the physical processes that are captured and their interaction with STAR-CCM+. These physical models give the MPC the capability to simulate the coupled physical processes that occur in SOFCs and other applications of interest to LGFCS.

The MPC has been validated against a wide range of SOFC experimental data obtained by LGFCS from single cell up to bundle scale over a broad range of test conditions. Each of the validation exercises is described in detail in terms of the model set up, numerical results and error analysis. In addition, the predictive capabilities of the MPC have also been tested in a variety of advanced engineering applications and the results from these have been presented. Feedback from these applications has been used to further improve the MPC modeling capabilities and computational methodology.

A key functional MPC requirement is the need to develop a simulation tool suitable for use by engineering staff outside of the project team. This was achieved by the incorporation of extensive user feedback into the GUI and the preparation of a user manual, tutorial exercises and training classes. The MPC has eight users who are involved in a wide range of simulation activities that includes SOFC performance analyses and SOFC related component applications. The MPC continues to be improved in response to staff requests arising from new applications.

The development of the MPC during this project has provided LGFCS with a level of analysis detail that had not previously been achieved using in-house modeling tools. The predictive capabilities of the MPC have been used to analyze a wide range of engineering applications and have made a significant contribution to the LGFCS SOFC program. The MPC is now in use by engineering staff for many different simulation applications and its usage is expected to expand in the future. 


\section{LIST OF FIGURES}

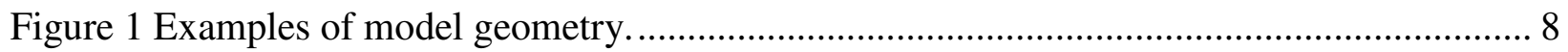

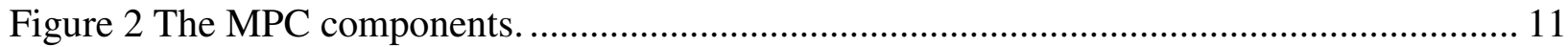

Figure 3 A typical view from the Excel model spreadsheet (EMS) ………………………...... 12

Figure 4 A typical view of the GUI installed into STAR-CCM+............................................... 13

Figure 5 Three layer model for analytical current flow solution............................................. 21

Figure 6 Variation of potential through cell layers.................................................................. 23

Figure 7 One-dimensional model of cell components ............................................................ 30

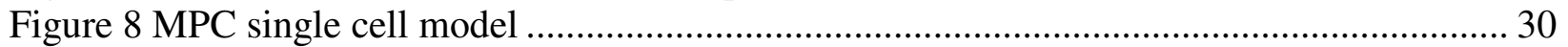

Figure 9 Hydrogen mole fraction distribution in the fuel side cell components. .......................... 31

Figure 10 Pressure distribution in the fuel side cell components. .............................................. 32

Figure 11 IV curve comparison between MPC single cell model predictions and experimental

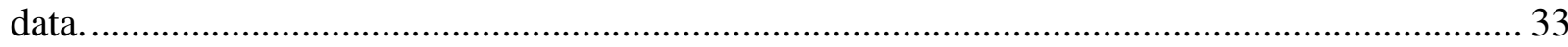

Figure 12 Calculated $E_{\text {cell }}(0)$ error magnitude for all 31 test cases.............................................. 34

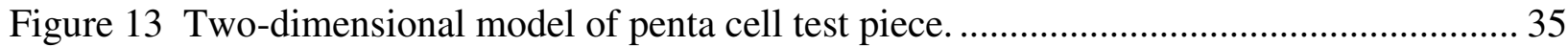

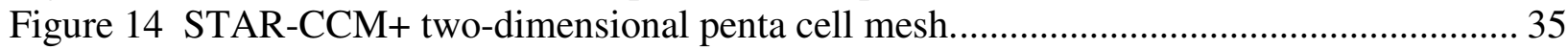

Figure 15 Hydrogen mole fraction distribution in the fuel side cell components. ....................... 36

Figure 16 Carbon monoxide mole fraction distribution in the fuel side model components. ...... 37

Figure 17 Current density distribution in each electrolyte of the penta cell model...................... 37

Figure 18 IV curve comparison between MPC penta cell model predictions and experimental

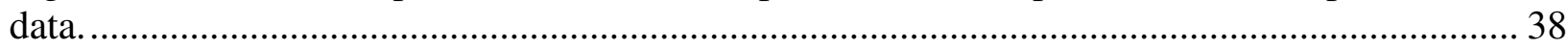

Figure 19 Calculated $E_{\text {cell }}(0)$ error magnitude for all 34 test cases............................................. 39

Figure 20 Thee-dimensional model of substrate.................................................................... 40

Figure 21 Close up of a STAR-CCM+ substrate model mesh...................................................... 40

Figure 22 Hydrogen mole fraction distribution in the fuel side cell components. ....................... 41

Figure 23 Calculated current density distribution in each electrolyte of a substrate................... 42

Figure 24 Thee-dimensional model of bundle ........................................................................... 42

Figure 25 Hydrogen mole fraction distribution in the fuel side bundle components .................... 43

Figure 26 Calculated $E_{\text {cell }}(0)$ error magnitude for all 48 test cases.............................................. 44

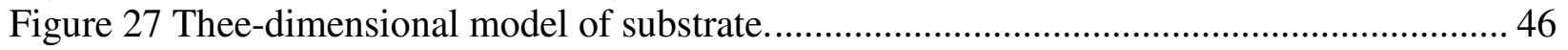

Figure 28 Close up of a STAR-CCM+ substrate model ............................................................. 46

Figure 29 Steady state distribution of hydrogen mole fraction within a three-dimensional

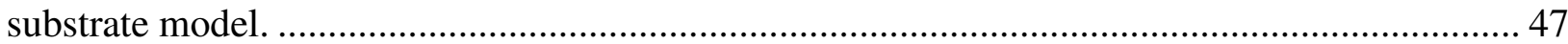

Figure 30 Transient distribution of hydrogen mole fraction 0.1 seconds after halving inlet fuel

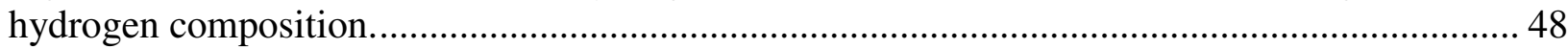

Figure 31 Transient distribution of hydrogen mole fraction 0.2 seconds after halving inlet fuel

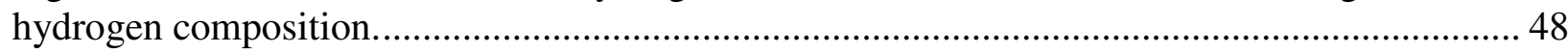

Figure 32 Two-dimensional model of five cell test piece................................................... 48

Figure 33 Voltage response of cells to sinusoidal input current signal ..................................... 49

Figure 34 Anode/Electrolyte hydrogen mole fraction response to sinusoidal input current signal.

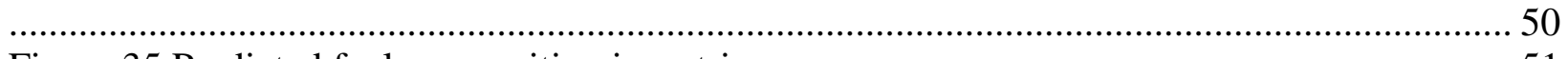

Figure 35 Predicted fuel composition in a strip....................................................................... 51

Figure 36 A simplified model of two bundles. ………...................................................... 52 


\section{REFERENCES}

[1] J.B. Young and B. Todd. Modeling of multi-component gas flows in capillaries and porous solids. Int. J. Heat Mass Transfer, 48, 5338-5353, 2005.

[2] B.A. Haberman. Three-Dimensional Simulation of the Integrated-Planar Solid Oxide Fuel Cell. $\mathrm{PhD}$ thesis, University of Cambridge, Cambridge, U.K., 2005.

[3] www.netbeans.org.

[4] B.A. Haberman and A.J Marquis. The Current Density Distribution in a Segmented-in-Series SOFC. ASME J Fuel Cell Sci. Tech., 6, 2009.

[5] J. Nielsen, M. Mogensen. SOFC LSM:YSZ cathode degradation induced by moisture: An impedance spectroscopy study. Solid State Ionics, 189, 74-81, 2011

\section{LIST OF ACRONYMS AND ABBREVIATIONS}

$\begin{array}{ll}\text { API } & \text { Application Programming Interface } \\ \text { CAD } & \text { Computer Aided Design } \\ \text { CFD } & \text { Computational Fluid Dynamics } \\ \text { CPIM } & \text { Cylindrical Pore Interpolation Model } \\ \text { DOE } & \text { U. S. Department of Energy } \\ \text { GUI } & \text { Graphical User Interface } \\ \text { MPC } & \text { Multi-physics Code } \\ \text { LGFCS } & \text { LG Fuel Cell Systems } \\ \text { SOFC } & \text { Solid Oxide Fuel Cell }\end{array}$

